\title{
A SAINT-VENANT PRINCIPLE FOR A THEORY OF NONLINEAR PLANE ELASTICITY
}

\author{
BY \\ C. O. HORGAN (University of Virginia, Charlottesville, Virginia) \\ AND \\ L. E. PAYNE (Cornell University, Ithaca, New York)
}

\begin{abstract}
A formulation of Saint-Venant's principle within the context of a restricted theory of nonlinear plane elasticity is described. The theory assumes small displacement gradients while the stress-strain relations are nonlinear. We consider plane deformations of such an elastic material occupying a rectangular region. The lateral sides are traction-free while the far end is subjected to a uniformly distributed tensile traction $\tau \geq 0$. The near end is subjected to a prescribed normal and shear traction. If this end loading were also that of uniform tension, then one possible corresponding stress state throughout the rectangle is that of uniform tension. When the near end loading is not uniform, the resulting stress field is expected to approach a uniform tensile state with increasing distance from the near end. This result is established here using differential inequality techniques for quadratic functionals. It is shown that, under certain constitutive assumptions, an energy-like quadratic functional, defined on the difference between the deformation field and the uniform tensile state, decays exponentially with distance from the near end. The estimated decay rate (which is a lower bound on the actual rate of decay) is characterized in terms of the load level $\tau$, the domain geometry, and material properties. The results predict a progressively slower decay of end effects with increasing load level $\tau$. The mathematical issues of concern involve spatial decay of solutions of a fourth-order nonlinear elliptic partial differential equation.
\end{abstract}

1. Introduction. The formulation and analysis of Saint-Venant's principle in $f$ nite elastostatics continues to attract attention. A version of Saint-Venant's principle within the framework of finite antiplane shear-the simplest possible setting within the theory of finite elasticity-was established in [1]. As was discussed in [1], the issues of concern in connection with Saint-Venant's principle in the nonlinear theory of elasticity are considerably more involved than those arising in the linearized theory. (For a survey of results on Saint-Venant's principle, primarily for the linearized theory, see, e.g., [2-5].) One difficulty is that the appropriate Saint-Venant solutions 
need to be carefully characterized (see, e.g., [6-13] and the references cited therein). Secondly, in the absence of superposition, consideration of self-equilibrated end loads is no longer sufficient. Furthermore, instabilities may have to be taken into account. Also the decay rate for end effects, even if exponential, might depend on the overall loading as well as on geometry and material characteristics. Several of these issues have been considered in studies in the nonlinear elasticity context [1, 14-27, 42] as well as in investigations of spatial decay of solutions of nonlinear elliptic partial differential equations [28-39]. The latter results may also be viewed as giving rise to principles of Phragmén-Lindelöf type.

The mathematical issues considered in [1] are concerned with the spatial decay of solutions of a Neumann boundary-value problem for a second-order quasilinear elliptic partial differential equation on a two-dimensional semi-infinite strip. The physical problem is that of finite antiplane shear of an isotropic nonlinearly elastic cylinder with a semi-infinite strip as cross-section. The long sides of the strip are traction free, while the near end is subjected to a prescribed shear traction. At infinity in the strip, the displacement is that of a simple shear with constant shear stress $\tau$ equal to the average shear stress generated at the near end. An estimate for the rate of exponential decay of stresses to this constant average value with distance from the end was obtained in [1]. The decay rate, which depends on the strip width and the strain-energy density of the material (as in the linear case), was also shown to be dependent on the overall loading of the strip, measured by $\tau$. The special case $\tau=0$ corresponds to the situation where the near end loading is self-equilibrated. This is the only case of interest in linearized elasticity. The results of [1] were obtained using arguments based on comparison (or maximum) principles for second-order quasilinear elliptic partial differential equations. An analog of the problem considered in [1] for a rectangular domain was investigated recently by the present authors [26] using an energy approach. Such methods, establishing the decay of certain quadratic functionals (i.e., functions of Lyapunov type) with distance from the loaded boundary, have been extensively used in investigations of Saint-Venant's principle (see, e.g., [2-5]). This approach is widely applicable in contrast to arguments based on the maximum principle which are generally limited to second-order problems (see, e.g., [33]).

The purpose of the present paper is to investigate similar issues in the context of a restricted theory of nonlinear plane elasticity. It is assumed that the displacement gradients are small so that the classical linearized strain measures are used while the stress-strain relations are nonlinear (such approximate theories have been widely used, for example, in plasticity theory). A Saint-Venant principle for another approximate theory of nonlinear two-dimensional elasticity has also been investigated recently by Breuer and Roseman [15], following on earlier work of Roseman [42]. See also $[19,21]$ for a treatment of Saint-Venant end effects within the theory of small deformations superimposed on large. In Secs. 2, 3 we formulate the boundaryvalue problem to be studied. We consider plane deformations of an elastic material occupying a rectangular region. The lateral sides are traction-free while the far end is subjected to a uniformly distributed tensile traction $\tau \geq 0$. The near end is subjected 
to a prescribed normal and shear traction. If this end loading were also that of uniform tension, then one possible corresponding stress state throughout the rectangle is that of uniform tension throughout. When the near end loading is not uniform, the resulting stress field is expected to approach a uniform tensile state with increasing distance from the near end, and this is the issue investigated here. It is convenient to formulate the basic problem using an Airy stress function approach. This formulation is described in Sec. 2 together with constitutive equations relating stresses and linearized strains. This leads, in Secs. 2, 3, to a boundary value problem for a single fourth-order nonlinear partial differential equation. The main results of the paper are established in Sec. 4. It is shown there that, under certain constitutive assumptions, an energy-like quadratic functional, defined on the difference between the deformation field and the uniform tensile state, decays exponentially with distance from the near end. The estimated decay rate (which is a lower bound for the actual rate of exponential decay) is characterized in terms of the load level $\tau$, the domain geometry, and material properties. In Sec. 5 an illustrative example is considered and the special case of self-equilibrated loading $(\tau=0)$ is examined. For the class of materials considered in this paper, results similar to those obtained in $[1,26]$ for hardening materials in antiplane shear are obtained. In particular, the results predict a progressively slower decay of end effects with increasing load level $\tau$. Some extensions of the main results are briefly outlined in Sec. 6 .

2. Plane deformation of a rectangular region. We are concerned with plane deformations of an elastic material occupying the rectangular region $R=\left\{\left(x_{1}, x_{2}\right) \mid 0<\right.$ $\left.x_{1}<l, 0<x_{2}<h\right\}, l \gg h$, where $\left(x_{1}, x_{2}\right)$ are rectangular Cartesian coordinates. The long sides of the rectangle are traction-free, the far end $x_{1}=l$ is subjected to a uniformly distributed tensile stress $\tau \geq 0$ while the end $x_{1}=0$ is subjected to a prescribed normal and shear traction. Thus, denoting the stress components by $\tau_{\alpha \beta}\left(x_{1}, x_{2}\right)$, where $\tau_{\alpha \beta}=\tau_{\beta \alpha}$, we have

$$
\begin{aligned}
& \tau_{12}\left(x_{1}, 0\right)=\tau_{22}\left(x_{1}, 0\right)=0, \quad \tau_{12}\left(x_{1}, h\right)=\tau_{22}\left(x_{1}, h\right)=0, \quad 0<x_{1}<l, \\
& \tau_{11}\left(l, x_{2}\right)=\tau, \quad \tau_{12}\left(l, x_{2}\right)=0, \quad 0 \leq x_{2} \leq h, \\
& \tau_{11}\left(0, x_{2}\right)=f\left(x_{2}\right), \quad \tau_{12}\left(0, x_{2}\right)=g\left(x_{2}\right), \quad 0 \leq x_{2} \leq h,
\end{aligned}
$$

where the given constant $\tau$ is nonnegative and the prescribed functions $f, g$ are continuous on $[0, h]$ and, to ensure continuity of shear tractions on the boundary, it is assumed that

$$
g(0)=g(h)=0 .
$$

The equilibrium equations, in the absence of body forces, are

$$
\tau_{\alpha \beta, \beta}=0 \text { in } R \text {. }
$$

The usual Cartesian tensor notation is used here and henceforth, with a repeated Greek subscript indicating summation from 1 to 2 and a subscript preceded by a comma denoting partial differentiation with respect to the corresponding coordinate. Equilibrium, together with the boundary conditions $(2.1)-(2.3)$, require that 
the boundary data $f, g$ satisfy the conditions

$$
\begin{gathered}
\int_{0}^{h} f\left(x_{2}\right) d x_{2}=\tau h, \quad \int_{0}^{h} x_{2} f\left(x_{2}\right) d x_{2}=\tau h^{2} / 2, \\
\int_{0}^{h} g\left(x_{2}\right) d x_{2}=0 .
\end{gathered}
$$

If $f\left(x_{2}\right)$ were constant on $[0, h]$ (and thus by $(2.6), f=\tau$ ) and $g \equiv 0$ on $[0, h]$ then (2.6), (2.7) would clearly be satisfied and the state of uniform tension

$$
\tau_{11}=\tau, \quad \tau_{22}=0, \quad \tau_{12}=0 \quad \text { on } R,
$$

would satisfy the equilibrium equations (2.5) and the boundary conditions (2.1)(2.3). When $f$ is not constant, one anticipates that the actual stress field might approach the state of uniform tension $(2.8)$ as $x_{1}$ increases. The principle of SaintVenant type established here quantifies this expectation. We note that when $\tau=0$, so that by (2.2) the far end of the rectangle is traction-free, the conditions (2.6), (2.7) express the fact that the loads on the near end must be self-equilibrated. By superposition, this is the situation of interest in the linearized theory (see, e.g., [2]).

The equilibrium equations (2.5), together with the boundary conditions (2.1)(2.3), do not constitute a complete statement of a boundary-value problem in twodimensional elasticity for the stresses $\tau_{\alpha \beta}$. We have three unknown stresses $\tau_{\alpha \beta}$ and two equations in (2.5). The third differential equation arises from constitutive and compatibility considerations. In what follows we describe a formulation of the boundary-value problem of concern in terms of a single stress function. First we introduce an Airy stress function $\phi\left(x_{1}, x_{2}\right)$ in terms of which the stresses are given by

$$
\tau_{11}=\phi_{.22}, \quad \tau_{22}=\phi_{.11}, \quad \tau_{12}=-\phi_{.12} .
$$

Equations (2.5) are then seen to be automatically satisfied, assuming that $\phi$ is sufficiently smooth to allow for interchange of orders of partial differentiation.

In the linearized theory of elasticity, the linearized strains are given by

$$
e_{\alpha \beta}=\frac{1}{2}\left(u_{\alpha, \beta}+u_{\beta, \alpha}\right) \text {, }
$$

where $u_{r \gamma}\left(x_{1}, x_{2}\right)$ are the components of displacement. The strains $e_{r \alpha}$ given by (2.10) must satisfy the compatibility condition

$$
e_{11.22}+e_{22.11}-2 e_{12.12}=0 .
$$

For a homogeneous isotropic linearly elastic solid, the in-plane stresses $\tau_{\alpha \beta}$ and strains $e_{\alpha \beta}$ in plane strain are related by

$$
e_{\alpha \beta}=\frac{(1+\nu)}{E} \tau_{\alpha \beta}-\frac{\nu}{E} \tau_{\%,} \delta_{\alpha \beta},
$$

where $E$ and $\nu$ are elastic constants. Substituting (2.9) in (2.12) and then in (2.11). one arrives at the familiar biharmonic equation of the linearized theory.

The nonlinear constitutive model of concern in what follows also involves the linearized strains (2.10). To motivate our considerations below, consider first the 
stress-strain relation given by

$$
e_{\alpha \beta}=\frac{(1+\nu)}{E} \tau_{\alpha \beta}-\frac{\nu}{E} \tau_{\gamma \gamma} \delta_{\alpha \beta}+\frac{1}{E} H\left(q^{2}\right) \tau_{\alpha \beta}, \quad q^{2}=\tau_{\alpha \beta} \tau_{\alpha \beta},
$$

where $E$ and $\nu$ are elastic constants, and $H\left(q^{2}\right) \geq 0$. The third term in (2.13) may be considered as a nonlinear correction to the linearized relation (2.12). Constitutive models of this type are widely used in plasticity theory. Substituting (2.9) in (2.13) and then in (2.11), one finds that $\phi$ must satisfy the fourth-order nonlinear partial differential equation

$$
\left[\rho\left(q^{2}\right) \phi_{, \alpha \beta}\right]_{, \alpha \beta}=0 \text { in } R,
$$

where

$$
\rho\left(q^{2}\right)=1+H\left(q^{2}\right), \quad q^{2}=\phi_{, \alpha \beta} \phi_{, \alpha \beta} .
$$

In the foregoing, the function $H$ is assumed to be a sufficiently smooth function of its indicated argument. When $H=0$ in (2.15), we have $\rho=1$ and (2.14) reduces to the biharmonic equation.

A more general model than (2.13) for a homogeneous isotropic elastic material also gives rise to the partial differential equation (2.14). In this model, we assume the existence of a strain-energy density function $W=W\left(e^{2}\right) \quad\left(e^{2} \equiv e_{\alpha \beta} e_{\alpha \beta}\right)$, for which

$$
\tau_{\alpha \beta}=\frac{\partial W}{\partial e_{\alpha \beta}}
$$

so that

$$
\tau_{\alpha \beta}=2 W^{\prime} e_{\alpha \beta}
$$

where the prime on $W$ denotes a derivative with respect to its argument. Substituting (2.9) in (2.17) and then in (2.11), we find that $\phi$ must satisfy the equation

$$
\left.\left[W^{\prime}\right)^{-1} \phi_{, \alpha \beta}\right]_{, \alpha \beta}=0 \text {. }
$$

Since (2.17), (2.9) imply that

$$
4 e^{2}\left[W^{\prime}\left(e^{2}\right)\right]^{2}=\phi_{, \alpha \beta} \phi_{, \alpha \beta} \equiv q^{2},
$$

we obtain, on assuming the invertibility of (2.19), that for some function $\mathscr{F}$

$$
e^{2}=\mathscr{F}\left(q^{2}\right) \text {. }
$$

Equation (2.18) has precisely the form of (2.14), on setting

$$
\rho\left(q^{2}\right)=\left[W^{\prime}\left(\mathscr{F}\left(q^{2}\right)\right)\right]^{-1} .
$$

The fourth-order nonlinear partial differential equation (2.14) has thus been shown to arise in two different constitutive settings. While these are subject to the limitation of small displacement gradients, the formulation of the present problem (and indeed the general plane problem) in terms of a single function $\phi$ satisfying (2.14), rather than in terms of three stresses satisfying a coupled system of partial differential equations, is of mathematical interest. 
It remains to express the boundary conditions (2.1)-(2.3) in terms of $\phi\left(x_{1}, x_{2}\right)$. On using (2.9), these boundary conditions can be written in terms of second derivatives of $\phi$. They can be integrated once and assuming continuity of $\phi_{, \alpha}$ on the boundary, can be shown (with the aid of $(2.6)_{1},(2.7)$ ) to reduce to

$$
\begin{gathered}
\phi_{, 1}\left(x_{1}, 0\right)=0, \quad \phi_{, 2}\left(x_{1}, 0\right)=0, \quad \phi_{, 1}\left(x_{1}, h\right)=0, \quad \phi_{, 2}\left(x_{1}, h\right)=\tau h, \\
0<x_{1}<l, \\
\phi_{, 1}\left(l, x_{2}\right)=0, \quad \phi_{, 2}\left(l, x_{2}\right)=\tau x_{2}, \quad 0 \leq x_{2} \leq h, \\
\phi_{, 2}\left(0, x_{2}\right)=\int_{0}^{x_{2}} f(s) d s, \quad \phi_{, 1}\left(0, x_{2}\right)=-\int_{0}^{x_{2}} g(s) d s, \quad 0 \leq x_{2} \leq h .
\end{gathered}
$$

On integrating (2.22)-(2.24) once more and assuming continuity of $\phi$ on the boundary, it can be shown, with the aid of $(2.6)_{2}$, that one obtains

$$
\begin{array}{lll}
\phi\left(x_{1}, 0\right)=0, & \phi_{.2}\left(x_{1}, 0\right)=0, & 0<x_{1}<l, \\
\phi\left(x_{1}, h\right)=\tau h^{2} / 2, & \phi_{.2}\left(x_{1}, h\right)=\tau h, & 0<x_{1}<l, \\
\phi\left(l, x_{2}\right)=\tau x_{2}^{2} / 2, & \phi_{.1}\left(l, x_{2}\right)=0, & 0 \leq x_{2} \leq h, \\
\phi\left(0, x_{2}\right)=F\left(x_{2}\right), & \phi_{.1}\left(0, x_{2}\right)=G\left(x_{2}\right), & 0 \leq x_{2} \leq h,
\end{array}
$$

where the prescribed functions $F, G$ are given by

$$
F\left(x_{2}\right)=\int_{0}^{x_{2}}\left\{\int_{0}^{t} f(s) d s\right\} d t, \quad G\left(x_{2}\right)=-\int_{0}^{x_{2}} g(s) d s .
$$

By virtue of (2.6), (2.7), it is seen that $F$ and $G$ must satisfy

$$
\begin{aligned}
& F(0)=0, \quad F(h)=\tau h^{2} / 2, \quad F^{\prime}(0)=0, \quad F^{\prime}(h)=\tau h, \\
& G(0)=0, \quad G(h)=0 .
\end{aligned}
$$

We observe that if $f\left(x_{2}\right)$ were constant on $[0, h]$ (so that $f=\tau$ ) and $g \equiv 0$ on $[0, h]$, we then would have $F \equiv \tau x_{2}^{2} / 2, G \equiv 0$, and the state of uniform tension (2.8) would correspond to

$$
\phi\left(x_{1}, x_{2}\right)=\tau x_{2}^{2} / 2 .
$$

It is readily verified that $\phi$ given by (2.32) would satisfy the differential equation (2.14) and the boundary conditions (2.25)-(2.28). When $f$ is not constant, we expect the solution of $(2.14),(2.25)-(2.28)$ to approach (2.32) as $x_{1}$ increases.

3. Statement of the boundary-value problem. The problem of plane deformations of the rectangular region $R$ under tensile loading has been shown to reduce to a boundary-value problem for the fourth-order partial differential equation (2.14), which for convenience is restated here. We are concerned with solutions $\phi\left(x_{1}, x_{2}\right)$ of the equation

$$
\left[\rho\left(q^{2}\right) \phi_{, \alpha \beta}\right]_{, \alpha \beta}=0 \text { in } R,
$$

where

$$
q^{2}=\phi_{, ; \delta} \phi, ; \delta,
$$


subject to the boundary conditions

$$
\begin{array}{lll}
\phi\left(x_{1}, 0\right)=0, & \phi_{, 2}\left(x_{1}, 0\right)=0, & 0<x_{1}<l, \\
\phi\left(x_{1}, h\right)=\tau h^{2} / 2, & \phi_{, 2}\left(x_{1}, h\right)=\tau h, & 0<x_{1}<l, \\
\phi\left(l, x_{2}\right)=\tau x_{2}^{2} / 2, & \phi_{, 1}\left(l, x_{2}\right)=0, & 0 \leq x_{2} \leq h, \\
\phi\left(0, x_{2}\right)=F\left(x_{2}\right), & \phi_{, 1}\left(0, x_{2}\right)=G\left(x_{2}\right), & 0 \leq x_{2} \leq h .
\end{array}
$$

The prescribed functions $F, G$ are such that

$$
\begin{array}{ccc}
F(0)=0, \quad F(h)=\tau h^{2} / 2, & F^{\prime}(0)=0, \quad F^{\prime}(h)=\tau h, \\
G(0)=0, & G(h)=0 .
\end{array}
$$

We assume the existence of solutions $\phi\left(x_{1}, x_{2}\right)$ of $(3.1)-(3.8)$ that are four times continuously differentiable in the interior of $R$ and twice continuously differentiable on its boundary.

4. Decay estimates for a quadratic functional. In this section, we establish our main decay estimate for solutions of (3.1)-(3.8). It is shown that the quadratic functional

$$
E(z)=\int_{R_{z}}\left[\rho\left(q^{2}\right)+\rho\left(\tau^{2}\right)\right] \psi_{, \alpha \beta} \psi_{, \alpha \beta} d A \quad\left(q^{2}=\phi_{, \alpha \beta} \phi_{, \alpha \beta}\right)
$$

contained in the subdomain $R_{z}=\left\{\left(x_{1}, x_{2}\right) \mid 0 \leq z<x_{1}<l, 0<x_{2}<h\right\}$ decays exponentially in $z$, where the function $\psi\left(x_{1}, x_{2}\right)$ is defined by

$$
\psi\left(x_{1}, x_{2}\right)=\phi\left(x_{1}, x_{2}\right)-\tau x_{2}^{2} / 2 \text {. }
$$

Thus $E(z)$ can be viewed as a "weighted energy" associated with the difference between $\phi\left(x_{1}, x_{2}\right)$ and the state of uniform tension (2.32).

The hypotheses that we make concerning $\rho$ are as follows. It is assumed that there exist positive constants $m$ and $M$, and a nonnegative constant $K$ such that, for all solutions of (3.1)-(3.8) we have

$$
0<m \leq \rho \leq M+K\left(\rho q^{2}\right)^{1-\delta}, \quad q^{2}=\phi_{, \alpha \beta} \phi_{, \alpha \beta},
$$

where $\delta$ is a constant such that

$$
0<\delta<1 .
$$

Furthermore, it is assumed that for a fixed value of the argument $s$ and for arbitrary values of $t$

$$
-\left[\rho\left(s^{2}\right)-\rho\left(t^{2}\right)\right]\left(s^{2}-t^{2}\right) \leq \sigma\left[\rho\left(s^{2}\right)+\rho\left(t^{2}\right)\right](s-t)^{2} \text { for some function } \sigma(t)<1 .
$$

Assumption (4.3) provides a restriction on the behavior of $\rho$ for small and large values of $q^{2}$. Without some such restrictions, one would not expect exponential decay of $\psi\left(x_{1}, x_{2}\right)$ defined by (4.2). If $\rho$ were a bounded function of its arguments, then $K$ in (4.3) could be taken to be zero. A function $\rho$ for which (4.3) holds is

$$
\rho=1+q^{2} \text {. }
$$

In this case, we may take $m=1, M=1, K=1$, and $\delta=\frac{1}{2}$. The function $\sigma(t)$ in the hypothesis (4.5) can be taken to be zero when $\rho$ is a monotone nondecreasing 
function of its argument since the left-hand side of (4.5) is then always nonpositive. Thus, in particular, when $\rho$ is given by (4.6), we may take $\sigma(t) \equiv 0$. It should be noted that (4.3), (4.5) do not imply that Eq. (3.1) is elliptic, although this is the case of primary interest.

We now proceed to establish our main result, namely that

$$
E(z) \leq K_{1} e^{-2 k z}, \quad 0 \leq z \leq l .
$$

Here

$$
k=k^{*}(\tau) / h
$$

is a function of $\tau$ (to be determined) which depends on the strain-energy density. We call $2 k$ the estimated rate of energy decay; it provides a lower bound for the actual rate of decay. The constant

$$
K_{1}=K_{1}\left(E_{0}, m, M, K, \delta, \sigma\right)
$$

depends on the quantity $E_{0} \equiv E(0)$, which is the total energy contained in the rectangle $R$. It is shown in Appendix B how an upper bound for $K_{1}$ in terms of geometry, boundary data, and strain-energy density of the material may be obtained.

To establish (4.7), we first derive the integro-differential inequality

$$
E+\gamma \int_{z}^{l} E(s) d s \leq A\left(-E^{\prime}+\gamma E\right)+B\left(-E^{\prime}+\gamma E\right)^{2},
$$

where $A, B$, and $\gamma$ are positive constants, and the prime denotes differentiation with respect to $z$. Inequality (4.10) will be shown to hold when the constant $K$ in (4.3) is positive. The result (4.7) is then obtained on integration of (4.10). When $K=0$, the result (4.7) will be derived in a different way.

Denoting by $L_{z}$ the line segment $x_{1}=z, 0 \leq x_{2} \leq h$, we find, with the help of the divergence theorem and (3.3)-(3.5), (4.2), that

$$
\begin{aligned}
E(z)= & -\int_{L_{z}}\left[\rho\left(q^{2}\right)+\rho\left(\tau^{2}\right)\right] \psi_{, 1 \alpha} \psi_{, \alpha} d x_{2}+\int_{L_{z}}\left\{\left[\rho\left(q^{2}\right)+\rho\left(\tau^{2}\right)\right] \psi_{, 1 \beta}\right\}_{, \beta} \psi d x_{2} \\
& +\int_{R_{z}}\left\{\left[\rho\left(q^{2}\right)+\rho\left(\tau^{2}\right)\right] \psi_{, \alpha \beta}\right\}_{, \alpha \beta} \psi d A .
\end{aligned}
$$

On using the differential equation (3.1), (4.2), and the divergence theorem together with (3.3)-(3.5), we may write (4.11) as

$$
\begin{aligned}
E(z)= & -\int_{L_{z}}\left[\rho\left(q^{2}\right) \phi_{, 1 \alpha}-\rho\left(\tau^{2}\right) v_{, 1 \alpha}\right] \psi_{, \alpha} d x_{2}+\int_{L_{z}}\left[\rho\left(q^{2}\right) \phi_{, 1 \beta}-\rho\left(\tau^{2}\right) v_{, 1 \beta}\right]_{, \beta} \psi d x_{2} \\
& +\frac{1}{2} \int_{R_{z}}\left[\rho\left(q^{2}\right)+\rho\left(\tau^{2}\right)\right] \psi_{, \alpha \beta} \psi_{, \alpha \beta} d A-\frac{1}{2} \int_{R_{z}}\left[\rho\left(q^{2}\right)-\rho\left(\tau^{2}\right)\right]\left(q^{2}-\tau^{2}\right) d A,
\end{aligned}
$$

where we have introduced the notation

$$
v\left(x_{1}, x_{2}\right)=\tau x_{2}^{2} / 2 \equiv v\left(x_{2}\right) .
$$


Employing the hypothesis (4.5) with $s=q, t=\tau$ to obtain an upper bound for the last integral on the right in (4.12), we then find

$$
\begin{aligned}
E(z) \leq-\int_{L_{z}} & {\left[\rho\left(q^{2}\right) \phi_{, 1 \alpha}-\rho\left(\tau^{2}\right) v_{, 1 \alpha}\right] \psi_{, \alpha} d x_{2} } \\
& +\int_{L_{z}}\left[\rho\left(q^{2}\right) \phi_{, 1 \beta}-\rho\left(\tau^{2}\right) v_{, 1 \beta}\right]_{, \beta} \psi d x_{2}+E(z) / 2 \\
& +(\sigma / 2) \int_{R_{z}}\left[\rho\left(q^{2}\right)+\rho\left(\tau^{2}\right)\right](q-\tau)^{2} d A,
\end{aligned}
$$

where the definition (4.1) has also been used. Now

$|q-\tau|=\left(q^{2}-2 \tau q+\tau^{2}\right)^{1 / 2} \leq\left(\phi_{, \alpha \beta} \phi_{, \alpha \beta}-2 v_{, \alpha \beta} \phi_{, \alpha \beta}+v_{, \alpha \beta} v_{, \alpha \beta}\right)^{1 / 2}=\left(\psi_{, \alpha \beta} \psi_{, \alpha \beta}\right)^{1 / 2}$,

by virtue of the facts that

$$
\tau^{2}=v_{, \alpha \beta} v_{, \alpha \beta}, \quad \tau q=\left(v_{, \alpha \beta} v_{, \alpha \beta}\right)^{1 / 2}\left(\phi_{, \gamma \delta} \phi_{, \gamma \delta}\right)^{1 / 2} \geq v_{, \alpha \beta} \phi_{, \alpha \beta} .
$$

Recalling (4.1), (4.13), we thus find from (4.14) that

$$
E(z)(1-\sigma) / 2 \leq-\int_{L_{z}} \rho\left(q^{2}\right) \phi_{, 1 \alpha} \psi_{, \alpha} d x_{2}+\int_{L_{z}}\left[\rho\left(q^{2}\right) \phi_{, 1 \beta}\right]_{, \beta} \psi d x_{2} .
$$

An integration of (4.17) leads to

$$
\left(\frac{1-\sigma}{2}\right) \int_{z}^{l} E(s) d s \leq-\int_{R_{z}} \rho\left(q^{2}\right) \phi_{, 1 \alpha} \psi_{, \alpha} d A+\int_{R_{z}}\left[\rho\left(q^{2}\right) \phi_{, 1 \beta}\right]_{, \beta} \psi d A,
$$

which can be written as

$$
\left(\frac{1-\sigma}{2}\right) \int_{z}^{l} E(s) d s \leq-\int_{L_{z}} \rho\left(q^{2}\right) \phi_{, 11} \psi d x_{2}-2 \int_{R_{z}} \rho\left(q^{2}\right) \phi_{, 1 \alpha} \psi_{, \alpha} d A,
$$

on using the divergence theorem and the boundary conditions (3.3)-(3.5) together with (4.2). From (4.19) we obtain

$$
\begin{aligned}
\left(\frac{1-\sigma}{2}\right) \int_{z}^{l} E(s) d s \leq & \int_{L_{z}} \rho\left(q^{2}\right)\left|\phi_{, 11}\right||\psi| d x_{2} \\
& +2 \int_{R_{z}} \rho\left(q^{2}\right)\left[\phi_{, 1 \alpha} \phi_{, 1 \alpha}\right]^{1 / 2}\left[\psi_{, \beta} \psi_{, \beta}\right]^{1 / 2} d A \\
\equiv & J_{1}+2 J_{2} .
\end{aligned}
$$

The hypothesis (4.3) is now used to obtain upper bounds for the integrals $J_{1}$ and $J_{2}$ in terms of $E(z)$ and $E^{\prime}(z)$. We find, in fact, that

$$
\begin{aligned}
J_{2} \leq & \int_{R_{z}}\left[M+K\left(\rho q^{2}\right)^{1-\delta}\right]^{1 / 2} \rho^{1 / 2}\left(\phi_{, 1 \alpha} \phi_{, 1 \alpha}\right)^{1 / 2}\left(\psi_{, \beta} \psi_{, \beta}\right)^{1 / 2} d A \\
\leq & M^{1 / 2} \int_{R_{z}}\left(\rho \phi_{, 1 \alpha} \phi_{, 1 \alpha}\right)^{1 / 2}\left(\psi_{, \beta} \psi_{, \beta}\right)^{1 / 2} d A \\
& +K^{1 / 2} \int_{R_{z}}\left(\rho q^{2}\right)^{(1-\delta) / 2} \rho^{1 / 2}\left(\phi_{, 1 \alpha} \phi_{, 1 \alpha}\right)^{1 / 2}\left(\psi_{, \beta} \psi_{, \beta}\right)^{1 / 2} d A
\end{aligned}
$$


Using (4.2) we now note that

$$
\left(\rho q^{2}\right)^{1 / 2} \leq\left(2 \rho \psi_{, \alpha \beta} \psi_{, \alpha \beta}\right)^{1 / 2}+\left(2 \rho \tau^{2}\right)^{1 / 2},
$$

and so

$$
\begin{aligned}
\left(\rho q^{2}\right)^{(1-\delta) / 2} & \leq\left[\left(2 \rho \psi_{, \alpha \beta} \psi_{, \alpha \beta}\right)^{1 / 2}+\left(2 \rho \tau^{2}\right)^{1 / 2}\right]^{1-\delta} \\
& \leq\left(2 \rho \psi_{, \alpha \beta} \psi_{, \alpha \beta}\right)^{(1-\delta) / 2}+\left(2 \rho \tau^{2}\right)^{(1-\delta) / 2} .
\end{aligned}
$$

Also, from (4.2), we see that

$$
\phi_{, 1 \alpha} \phi_{, 1 \alpha}=\psi_{, 1 \alpha} \psi_{, 1 \alpha} \leq \psi_{, \alpha \beta} \psi_{, \alpha \beta} .
$$

Employing (4.24) and (4.25) in (4.22) we obtain

$$
\begin{aligned}
J_{2} \leq & M^{1 / 2} \int_{R_{z}}\left(\rho \psi_{, 1 \alpha} \psi_{, 1 \alpha}\right)^{1 / 2}\left(\psi_{, \beta} \psi_{, \beta}\right)^{1 / 2} d A \\
& +\left(2^{1-\delta} K\right)^{1 / 2} \int_{R_{z}}\left(\rho \psi_{, \alpha \beta} \psi_{, \alpha \beta}\right)^{1-\delta / 2}\left(\psi_{, \gamma} \psi_{, \gamma}\right)^{1 / 2} d A \\
& +\left(2^{1-\delta} K\right)^{1 / 2} \tau^{1-\delta} \int_{R_{z}}\left[\rho\left(\phi_{, 1,} \phi_{, 1 \alpha}\right)^{1 / 2}\left(\psi_{, \beta} \psi_{, \beta}\right)^{1 / 2}\right]^{1-\delta} \\
& \cdot\left[\rho^{1 / 2}\left(\phi_{, 1 \gamma} \phi_{, 1 \gamma}\right)^{1 / 2}\left(\psi_{, \mu} \psi_{, \mu}\right)^{1 / 2}\right]^{\delta} d A \\
\equiv & M^{1 / 2} I_{1}+\left(2^{1-\delta} K\right)^{1 / 2} I_{2}+\left(2^{1-\delta} K\right)^{1 / 2} \tau^{1-\delta} I_{3},
\end{aligned}
$$

where $\rho=\rho\left(q^{2}\right)$. By virtue of Schwarz's inequality,

$$
I_{1} \leq\left(\int_{R_{z}} \rho \psi_{,{ }_{1 \alpha}} \psi_{,{ }_{1 \alpha}} d A\right)^{1 / 2}\left(\int_{R_{z}} \psi_{, \beta} \psi_{, \beta} d A\right)^{1 / 2} .
$$

Now the boundary conditions (3.3), (3.4) and the definition (4.2) imply that

$$
\psi_{, 1}=0, \quad \psi_{, 2}=0 \quad \text { at } x_{2}=0, h, 0<x_{1}<l .
$$

Thus, by Wirtinger's inequality, we have

$$
\int_{L_{z}} \psi_{, \beta 2} \psi_{, \beta 2} d x_{2} \geq \lambda_{1} \int_{L_{z}} \psi_{, \beta} \psi_{, \beta} d x_{2},
$$

where

$$
\lambda_{1}=\pi^{2} / h^{2} \text {. }
$$

On insertion of (4.29) in (4.27) and recalling from (4.3) that $\rho \geq m$, we find that

$$
\begin{aligned}
I_{1} & \leq\left(\lambda_{1} m\right)^{-1 / 2}\left(\int_{R_{z}} \rho \psi_{, 1 \alpha} \psi_{, 1 \alpha} d A\right)^{1 / 2}\left(\int_{R_{z}} \rho \psi_{.2 \beta} \psi_{, 2 \beta} d A\right)^{1 / 2} \\
& \leq 2^{-1}\left(\lambda_{1} m\right)^{-1 / 2}\left(\int_{R_{z}} \rho \psi_{, \alpha \beta} \psi_{, \alpha \beta} d A\right)
\end{aligned}
$$

where the arithmetic-geometric mean inequality has been used to obtain (4.32) from (4.31). Recalling the definition of $E(z)$ from (4.1), we write (4.32) as

$$
I_{1} \leq 2^{-1}\left(\lambda_{1} m\right)^{-1 / 2} E(z) \text {. }
$$


To obtain an upper bound for $I_{2}$, we employ the Hölder inequality

$$
\int f g \leq\left(\int f^{p}\right)^{1 / p}\left(\int g^{q}\right)^{1 / q}, \quad \frac{1}{p}+\frac{1}{q}=1,
$$

with the choice $f=\left(\rho \psi_{, \alpha \beta} \psi_{, \alpha \beta}\right)^{1-\delta / 2}, g=\left(\psi_{, \gamma} \psi_{, \gamma}\right)^{1 / 2}, p=(1-\delta / 2)^{-1}, q=2 / \delta$, and so find that

$$
I_{2} \leq\left(\int_{R_{z}} \rho \psi_{, \alpha \beta} \psi_{, \alpha \beta} d A\right)^{1-\delta / 2}\left(\int_{R_{z}}\left(\psi_{, \gamma} \psi_{, \gamma}\right)^{1 / \delta} d A\right)^{\delta / 2} .
$$

Since $\psi_{, \alpha}=0$ on three sides of the rectangle $R_{z}$ by virtue of (4.2) and the boundary conditions $(3.3)-(3.5)$, we have a Sobolev inequality

$$
\left[\int_{R_{z}}\left(\psi_{, \gamma} \psi_{, \gamma}\right)^{1 / \delta} d A\right]^{\delta} \leq \frac{1}{\Omega(1 / \delta)} \int_{R_{z}} \psi_{, \alpha \beta} \psi_{, \alpha \beta} d A, \quad 0<\delta<1,
$$

where a Sobolev constant $\Omega(1 / \delta)$ can be found (see Appendix A) explicitly in terms of $\delta$ and $h$. On employing (4.36) in (4.35), and using $\rho \geq m$, we deduce that

$$
I_{2} \leq(\Omega m)^{-1 / 2} E(z)^{(3-\delta) / 2} .
$$

To obtain an upper bound for $I_{3}$, we make use of Hölder's inequality and the inequality (see, e.g., [40, p. 37])

$$
a^{s} b^{1-s} \leq s a+(1-s) b \quad(a, b \geq 0,0 \leq s \leq 1),
$$

to get

$$
\int(\alpha f)^{1-\delta}\left(\frac{g}{\alpha^{(1-\delta) / \delta}}\right)^{\delta} \leq(1-\delta) \alpha \int f+\frac{\delta}{\alpha^{(1-\delta) / \delta}} \int g,
$$

where $\alpha$ is an arbitrary positive constant. Choose $f=\rho\left(\phi_{, 1 \alpha} \phi_{, 1 \alpha}\right)^{1 / 2}\left(\psi_{, \beta} \psi_{, \beta}\right)^{1 / 2}$ and $g=\rho^{1 / 2}\left(\phi_{, 1 \gamma} \phi_{, 1 \gamma}\right)^{1 / 2}\left(\psi_{, \mu} \psi_{, \mu}\right)^{1 / 2}$ in (4.39). Thus we find that

$$
\begin{aligned}
I_{3} & \leq \alpha(1-\delta) J_{2}+\frac{\delta}{\alpha^{(1-\delta) / \delta}} I_{1} \\
& \leq \alpha(1-\delta) J_{2}+\frac{\delta E(z)}{2 \alpha^{(1-\delta) / \delta}\left(\lambda_{1} m\right)^{1 / 2}},
\end{aligned}
$$

where (4.33) has been used to obtain the final inequality in (4.40). On using (4.40), (4.37), and (4.33) in (4.26), we get

$$
\begin{aligned}
{\left[1-\alpha(1-\delta)\left(2^{1-\delta} K\right)^{1 / 2} \tau^{1-\delta}\right] J_{2} \leq } & {\left[M^{1 / 2}+\frac{\delta \tau^{1-\delta}\left(2^{1-\delta} K\right)^{1 / 2}}{\alpha^{(1-\delta) / \delta}}\right]\left(\lambda_{1} m\right)^{-1 / 2} \frac{E(z)}{2} } \\
& +\left(2^{1-\delta} K\right)^{1 / 2}(\Omega m)^{-1 / 2} E(z)^{(3-\delta) / 2}
\end{aligned}
$$

The choice

$$
\alpha=\left[2(1-\delta) \tau^{1-\delta}\left(2^{1-\delta} K\right)^{1 / 2}\right]^{-1}
$$


in (4.41) yields the upper bound

$$
\begin{aligned}
J_{2} \leq & \left(\lambda_{1} m\right)^{-1 / 2}\left[M^{1 / 2}+\left(2^{1-\delta} K\right)^{1 / 2} \delta \tau^{1-\delta}\left\{2(1-\delta) \tau^{1-\delta}\left(2^{1-\delta} K\right)^{1 / 2}\right\}^{(1-\delta) / \delta}\right] E(z) \\
& +2\left(2^{1-\delta} K\right)^{1 / 2}(\Omega m)^{-1 / 2} E(z)^{(3-\delta) / 2}
\end{aligned}
$$

Similar arguments are used to find an upper bound for $J_{1}$ defined in (4.20). Thus on employing (4.3) we find that

$$
\begin{aligned}
J_{1} & \leq \int_{L_{z}}\left[M+K\left(\rho q^{2}\right)^{1-\delta}\right]^{1 / 2} \rho^{1 / 2}\left|\phi_{, 11} \| \psi\right| d x_{2} \\
& \leq M^{1 / 2} \int_{L_{z}} \rho^{1 / 2}\left|\phi_{, 11} \| \psi\right| d x_{2}+\left(2^{1-\delta} K\right)^{1 / 2} \int_{L_{z}}\left(\rho q^{2}\right)^{(1-\delta) / 2} \rho^{1 / 2}\left|\phi_{, 11}\right||\psi| d x_{2} .
\end{aligned}
$$

We now use (4.24), (4.25) in (4.44) and the fact that $\left|\phi_{, 11}\right|=\left(\phi_{, 11} \phi_{, 11}\right)^{1 / 2}=$ $\left(\psi_{, 11} \psi_{, 11}\right)^{1 / 2} \leq\left(\psi_{, \alpha \beta} \psi_{, \alpha \beta}\right)^{1 / 2}$ to get

$$
\begin{aligned}
J_{1} \leq & M^{1 / 2} \int_{L_{z}} \rho^{1 / 2}\left|\phi_{, 11}\right||\psi| d x_{2}+\left(2^{1-\delta} K\right)^{1 / 2} \int_{L_{z}}\left(\rho \psi_{, \alpha \beta} \psi_{, \alpha \beta}\right)^{(1-\delta) / 2}|\psi| d x_{2} \\
& +\left(2^{1-\delta} K\right)^{1 / 2} \tau^{1-\delta} \int_{L_{z}}\left[\rho\left|\phi_{, 11}\right||\psi|\right]^{1-\delta}\left[\rho^{1 / 2}\left|\phi_{, 11} \| \psi\right|\right]^{\delta} d x_{2} \\
\equiv & M^{1 / 2} I_{4}+\left(2^{1-\delta} K\right)^{1 / 2} I_{5}+\left(2^{1-\delta} K\right)^{1 / 2} \tau^{1-\delta} I_{6} .
\end{aligned}
$$

By Schwarz's inequality

$$
\begin{aligned}
I_{4} & \leq\left(\int_{L_{z}} \rho \phi_{, 11}^{2} d x_{2}\right)^{1 / 2}\left(\int_{L_{z}} \psi^{2} d x_{2}\right)^{1 / 2} \\
& =\left(\int_{L_{z}} \rho \psi_{, 11}^{2} d x_{2}\right)^{1 / 2}\left(\int_{L_{z}} \psi^{2} d x_{2}\right)^{1 / 2},
\end{aligned}
$$

where (4.2) has been used to obtain (4.47) from (4.46). Since $\psi=0$ and $\psi_{, 2}=0$ at $x_{2}=0, h\left(0<x_{1}<l\right)$, we have the Wirtinger-type inequality

$$
\int_{L_{z}} \psi_{, 22}^{2} d x_{2} \geq \lambda_{2} \int_{L_{z}} \psi^{2} d x_{2}
$$

where

$$
\lambda_{2} \geq(3 \pi / 2 h)^{4} .
$$

On insertion of (4.48) in (4.47), and using $\rho \geq m$, we find that

$$
\begin{aligned}
I_{4} & \leq\left(\lambda_{2} m\right)^{-1 / 2}\left(\int_{L_{z}} \rho \phi_{, 11}^{2} d x_{2}\right)^{1 / 2}\left(\int_{L_{z}} \rho \psi_{, 22}^{2} d x_{2}\right)^{1 / 2} \\
& \leq 2^{-1}\left(\lambda_{2} m\right)^{-1 / 2}\left(\int_{L_{z}} \rho \psi_{, \alpha \beta} \psi_{, \alpha \beta} d x_{2}\right)
\end{aligned}
$$

and so, by virtue of (4.1), we have

$$
I_{4} \leq 2^{-1}\left(\lambda_{2} m\right)^{-1 / 2}\left[-E^{\prime}(z)\right] .
$$


To obtain an upper bound for $I_{5}$, we use the Hölder inequality (4.34) with the choice $f=\left(\rho \psi_{, \alpha \beta} \psi_{, \alpha \beta}\right)^{1-\delta / 2}, g=\left(\psi^{2}\right)^{1 / 2}, p=(1-\delta / 2)^{-1}, q=2 / \delta$ to get

$$
I_{5} \leq\left(\int_{L_{z}} \rho \psi_{, \alpha \beta} \psi_{, \alpha \beta} d x_{2}\right)^{1-\delta / 2}\left[\int_{L_{z}}\left(\psi^{2}\right)^{1 / \delta} d x_{2}\right]^{\delta / 2} .
$$

Since $\psi=0$ at the end points of $L_{z}$, we have a one-dimensional Sobolev inequality

$$
\left[\int_{L_{z}} \psi^{2 / \delta} d x_{2}\right]^{\delta} \leq \frac{1}{\omega(1 / \delta)} \int_{L_{z}} \psi_{, 2}^{2} d x_{2}, \quad 0<\delta<1,
$$

where a Sobolev constant $\omega(1 / \delta)$ can be found (see Appendix A) explicitly in terms of $\delta$ and $h$. Since $\psi=0$ and $\psi_{, 2}=0$ at the end points of $L_{z}$, we also have a Wirtinger-type inequality

$$
\int_{L_{z}} \psi_{, 22}^{2} d x_{2} \geq \lambda_{3} \int_{L_{z}} \psi_{, 2}^{2} d x_{2}
$$

where

$$
\lambda_{3}=4 \pi^{2} / h^{2}
$$

On using (4.55), (4.1), and $\rho \geq m$ in (4.53) we deduce that

$$
I_{5} \leq\left(\lambda_{3} \omega m\right)^{-1 / 2}\left[-E^{\prime}(z)\right]^{(3-\delta) / 2} .
$$

To obtain an upper bound for $I_{6}$, we use (4.39) with the choice $f=\rho|\phi, 11||\psi|$, $g=\rho^{1 / 2}|\phi, 11||\psi|$, and $\alpha=\beta$, an arbitrary positive constant. Thus we find that

$$
\begin{aligned}
I_{6} & \leq \beta(1-\delta) J_{1}+\frac{\delta}{\beta^{(1-\delta) / \delta}} I_{4} \\
& \leq \beta(1-\delta) J_{1}+\frac{\delta\left[-E^{\prime}(z)\right]}{2 \beta^{(1-\delta) / \delta}\left(\lambda_{2} m\right)^{1 / 2}} .
\end{aligned}
$$

Now using (4.59), (4.57), and (4.52) in (4.44) we find that

$$
\begin{aligned}
& {\left[1-\left(2^{1-\delta} K\right)^{1 / 2} \tau^{1-\delta}(1-\delta) \beta\right] J_{1}} \\
& \leq\left[M^{1 / 2}+\frac{\delta \tau^{1-\delta}\left(2^{1-\delta} K\right)^{1 / 2}}{\beta^{(1-\delta) / \delta}}\right]\left(\lambda_{2} m\right)^{-1 / 2}\left[-E^{\prime}(z)\right] / 2 \\
& \quad+\left(2^{1-\delta} K\right)^{1 / 2}\left(\lambda_{3} \omega m\right)^{-1 / 2}\left[-E^{\prime}(z)\right]^{(3-\delta) / 2} .
\end{aligned}
$$

On choosing

$$
\beta=\left[2(1-\delta) \tau^{1-\delta}\left(2^{1-\delta} K\right)^{1 / 2}\right]^{-1},
$$

we deduce from (4.60) the upper bound

$$
\begin{aligned}
J_{1} \leq & \left(\lambda_{2} m\right)^{-1 / 2}\left[M^{1 / 2}+\left(2^{1-\delta} K\right)^{1 / 2} \delta \tau^{1-\delta}\left\{2(1-\delta) \tau^{1-\delta}\left(2^{1-\delta} K\right)^{1 / 2}\right\}^{(1-\delta) / \delta}\right]\left[-E^{\prime}(z)\right] \\
& +2\left(2^{1-\delta} K\right)^{1 / 2}\left(\lambda_{3} \omega m\right)^{-1 / 2}\left[-E^{\prime}(z)\right]^{(3-\delta) / 2} .
\end{aligned}
$$


Finally, on substitution from (4.62) and (4.43) in (4.20) we obtain

$$
\begin{aligned}
\left(\frac{1-\sigma}{2}\right) \int_{z}^{l} E(s) d s \leq & C E(z)+D E(z)^{(3-\delta) / 2} \\
& +\left(\frac{\lambda_{1}}{4 \lambda_{2}}\right)^{1 / 2} C\left[-E^{\prime}(z)\right]+\left(\frac{\Omega}{4 \lambda_{3} \omega}\right)^{1 / 2} D\left[-E^{\prime}(z)\right]^{(3-\delta) / 2}
\end{aligned}
$$

where the constants $C$ and $D$ are given by

$$
\begin{gathered}
C=2\left(\lambda_{1} m\right)^{-1 / 2}\left[M^{1 / 2}+\left(2^{1-\delta} K\right)^{1 / 2} \delta \tau^{1-\delta}\left\{2(1-\delta) \tau^{1-\delta}\left(2^{1-\delta} K\right)^{1 / 2}\right\}^{(1-\delta) / \delta}\right] \\
D=4\left(2^{1-\delta} K\right)^{1 / 2}(\Omega m)^{-1 / 2}
\end{gathered}
$$

The integro-differential inequality (4.63) will now be shown to be equivalent to (4.10), whenever $K \neq 0$. The case when $K=0$ in (4.3) will be treated separately. For any positive function $F(z)$ we have

$$
F^{(3-\delta) / 2}=\left(F^{2}\right)^{(1-\delta) / 2} F^{(1+\delta) / 2}=\left(k_{1} F^{2}\right)^{(1-\delta) / 2}\left(\frac{F}{k_{1}^{(1-\delta) /(1+\delta)}}\right)^{(1+\delta) / 2},
$$

and so, on using the inequality (4.38), we find

$$
F^{(3-\delta) / 2} \leq\left(\frac{1-\delta}{2}\right) k_{1} F^{2}+\left(\frac{1+\delta}{2}\right) \frac{F}{k_{1}^{(1-\delta) /(1+\delta)}},
$$

where $k_{1}>0$ is an arbitrary constant. Employing the inequality (4.67) with $F$ chosen to be $E$ and $-E^{\prime}$ in turn (with constant $k_{2}>0$ ), we may write (4.63) as

$$
\begin{aligned}
k_{3} E(z) & +\left(\frac{1-\sigma}{2}\right) \int_{z}^{l} E(s) d s \\
\leq & {\left[C+\left(\frac{1+\delta}{2}\right) \frac{D}{k_{1}^{(1-\delta) /(1+\delta)}}+k_{3}\right] E(z) } \\
& +\frac{D(1-\delta)}{2} k_{1} E^{2}(z)+\left[\left(\frac{\lambda_{1}}{4 \lambda_{2}}\right)^{1 / 2} C+\frac{(1+\delta)}{2}\left(\frac{\Omega}{4 \lambda_{3} \omega}\right)^{1 / 2} \frac{D}{\left.k_{2}^{(1-\delta) /(1+\delta)}\right]\left[-E^{\prime}(z)\right]}\right. \\
& +D\left(\frac{1-\delta}{2}\right)\left(\frac{\Omega}{4 \lambda_{3} \omega}\right)^{1 / 2} k_{2}\left[-E^{\prime}(z)\right]^{2}
\end{aligned}
$$

where $k_{3}>0$ is an arbitrary constant. Let

$$
\begin{gathered}
k_{3}=\left(\frac{1-\sigma}{2}\right) \nu, \\
k_{2}=\nu^{2}\left(\frac{4 \lambda_{3} \omega}{\Omega}\right)^{1 / 2} k_{1},
\end{gathered}
$$


where $\nu>0$ is a constant. Then (4.68) can be written as

$$
\begin{aligned}
&\left(\frac{1-\sigma}{2}\right)\left\{\int_{z}^{l} E(s) d s+\nu E(z)\right\} \\
& \leq \frac{D(1-\delta)}{2} k_{1}\left[E^{2}(z)+\nu^{2}\left[E^{\prime}(z)\right]^{2}\right] \\
&+\left\{C+\frac{(1+\delta)}{2} \frac{D}{k_{1}^{(1-\delta) /(1+\delta)}}+\frac{(1-\sigma) \nu}{2}\right\} E(z) \\
&+\left\{\left(\frac{\lambda_{1}}{4 \lambda_{2}}\right)^{1 / 2} C+\frac{(1+\delta)}{2}\left(\frac{\Omega}{4 \lambda_{3} \omega}\right)^{1 /(1+\delta)} \frac{D}{\left(\nu^{2} k_{1}\right)^{(1-\delta) /(1+\delta)}}\right\}\left[-E^{\prime}(z)\right]
\end{aligned}
$$

We wish now to choose $k_{1}$ and $\nu$ in such a way that

$$
\begin{gathered}
\left\{\left(\frac{\lambda_{1}}{4 \lambda_{2}}\right)^{1 / 2} C+\frac{(1+\delta)}{2}\left(\frac{\Omega}{4 \lambda_{3} \omega}\right)^{1 /(1+\delta)} \frac{D}{\left(\nu^{2} k_{1}\right)^{(1-\delta) /(1+\delta)}}\right\} \\
\geq \nu\left\{C+\frac{(1+\delta) D}{2\left(k_{1}\right)^{(1-\delta) /(1+\delta)}}+\frac{(1-\sigma) \nu}{2}\right\} .
\end{gathered}
$$

To simplify this inequality, we replace the unknown constants $k_{1}$ and $\nu$ by new constants $\alpha$ and $\beta$ through the substitution

$$
D / k_{1}^{(1-\delta) /(1+\delta)}=\alpha C, \quad \nu=\beta C .
$$

Then (4.72) becomes

$$
\begin{gathered}
\left\{\left(\frac{\lambda_{1}}{4 \lambda_{2}}\right)^{1 / 2}+\frac{(1+\delta)}{2}\left(\frac{\Omega}{4 \lambda_{3} \omega}\right)^{1 /(1+\delta)} \frac{\alpha}{(\beta C)^{2(1-\delta) /(1+\delta)}}\right\} \\
\geq \beta C\left\{1+\frac{(1+\delta)}{2} \alpha+\frac{(1-\sigma)}{2} \beta\right\} .
\end{gathered}
$$

We note that if $K=0$ in (4.3), then (4.65) implies that $D=0$ and so from (4.73) we find $\alpha=0$. For $K \neq 0$, we observe that it is possible to satisfy (4.74) with the equality sign (for some admissible positive $\beta$ ) for given values of $\delta, h$, and for any choice of the positive constant $\alpha$. For simplicity, we shall choose $\alpha=1$ and choose $\beta$ to satisfy

$$
\left(\frac{\lambda_{1}}{4 \lambda_{2}}\right)^{1 / 2}=\beta C\left\{\frac{3+\delta}{2}+\frac{(1-\sigma)}{2} \beta\right\},
$$

so that (4.74) is satisfied with strict inequality. Thus $\beta$ is the positive root of the quadratic in (4.75) and is given as

$$
\beta=\beta_{*}=\frac{1}{2}\left\{-\left(\frac{3+\delta}{1-\sigma}\right)+\left[\left(\frac{3+\delta}{1-\sigma}\right)^{2}+\frac{8}{(1-\sigma) C}\left(\frac{\lambda_{1}}{4 \lambda_{2}}\right)^{1 / 2}\right]^{1 / 2}\right\}
$$


With this choice for $\beta$, together with (4.73), we find that (4.71) yields the inequality

$$
\begin{aligned}
\frac{(1-\sigma)}{2} & \left\{\int_{z}^{l} E(s) d s+\beta_{*} C E(z)\right\} \\
\leq & \frac{D(1-\delta)}{2}\left(\frac{D}{C}\right)^{(1+\delta) /(1-\delta)}\left\{E^{2}(z)+\beta_{*}^{2} C^{2}\left[E^{\prime}(z)\right]^{2}\right\} \\
& +\beta_{*}^{-1}\left\{\left(\frac{\lambda_{1}}{4 \lambda_{2}}\right)^{1 / 2}+\frac{(1+\delta)}{2}\left(\frac{\Omega}{4 \lambda_{3} \omega}\right)^{1 /(1+\delta)} \frac{1}{\left(\beta_{*} C\right)^{2(1-\delta) /(1+\delta)}}\right\} \\
& \times\left\{E(z)-\beta_{*} C E^{\prime}(z)\right\},
\end{aligned}
$$

which, in turn, leads to

$$
H(z) \leq A\left[-H^{\prime}(z)\right]+B\left[-H^{\prime}(z)\right]^{2},
$$

where

$$
\begin{gathered}
H(z)=E(z)+\left(\beta_{*} C\right)^{-1} \int_{z}^{l} E(s) d s \\
A=\frac{2}{(1-\sigma) \beta_{*}}\left\{\left(\frac{\lambda_{1}}{4 \lambda_{2}}\right)^{1 / 2}+\frac{(1+\delta)}{2}\left(\frac{\Omega}{4 \lambda_{3} \omega}\right)^{1 /(1+\delta)} \frac{1}{\left(\beta_{*} C\right)^{2(1-\delta) /(1+\delta)}}\right\}, \\
B=\frac{D(1-\delta)}{1-\sigma}\left(\frac{D}{C}\right)^{(1+\delta) /(1-\delta)} \beta_{*} C=\beta_{*}\left(\frac{1-\delta}{1-\sigma}\right) \frac{D^{2 /(1-\delta)}}{C^{2 \delta /(1-\delta)}} .
\end{gathered}
$$

The differential inequality (4.78) for $H(z)$ is equivalent to the integro-differential inequality (4.10) for $E(z)$, with $\gamma=\left(\beta_{*} C\right)^{-1}$.

Completing squares in (4.78) we find, for $B \neq 0$,

$$
\left(-H^{\prime}+A / 2 B\right)^{2} \geq B^{-1}\left(H+A^{2} / 4 B\right),
$$

so that

$$
-H^{\prime} \geq B^{-1 / 2}\left\{\left(H+A^{2} / 4 B\right)^{1 / 2}-A /\left(2 B^{1 / 2}\right)\right\} .
$$

On integration of (4.83) from 0 to $z$ we obtain

$$
-\int_{H(0)}^{H(z)} \frac{d \eta}{\left(\eta+d^{2}\right)^{1 / 2}-d} \geq \frac{z}{B^{1 / 2}},
$$

where

$$
d=A /\left(2 B^{1 / 2}\right) .
$$

The integral in (4.84) is readily evaluated to yield

$$
2\left[\left\{H(0)+d^{2}\right\}^{1 / 2}-\left\{H(z)+d^{2}\right\}^{1 / 2}\right]+2 d \ln \left[\frac{\left\{H(0)+d^{2}\right\}^{1 / 2}-d}{\left\{H(z)+d^{2}\right\}^{1 / 2}-d}\right] \geq z / B^{1 / 2} .
$$

We now show how (4.86) leads to the desired exponential decay estimate (4.7). The second term on the left-hand side of (4.86) may be dropped and then (4.86) leads to

$$
\left[H(z)+d^{2}\right]^{1 / 2}-d \leq\left[\left\{H(0)+d^{2}\right\}^{1 / 2}-d\right] \exp \left[\left\{H(0)+2 d^{2}\right\}^{1 / 2} / d\right] e^{-z / 2 d B^{1 / 2}}
$$


Multiplying both sides of $(4.87)$ by $\left[H(z)+d^{2}\right]^{1 / 2}+d$ and observing that (4.79) implies $H(z) \leq H(0)$, we deduce that

$$
H(z) \leq H(0) \exp \left[\left\{H(0)+2 d^{2}\right\}^{1 / 2} / d\right] e^{-z / 2 d B^{1 / 2}} .
$$

By virtue of its definition in (4.79) we have $H(z) \geq E(z)$ and so (4.88) yields

$$
E(z) \leq K_{1} e^{-2 k z}, \quad 0 \leq z \leq l,
$$

where the estimated decay rate $2 k$ is given by

$$
2 k=1 /\left(2 d B^{1 / 2}\right)=1 / A,
$$

and $A$ is given in (4.80). The multiplicative factor $K_{1}$ in (4.89) is

$$
K_{1}=H(0) \exp \left[\left\{H(0)+d^{2}\right\}^{1 / 2} / d\right],
$$

where $d$ is given by (4.85). This completes the derivation of the exponential decay estimate (4.7) whenever the constant $K$ in (4.3) is positive.

We remark here that there is an alternative way of proceeding from (4.86) to arrive at an estimate of the form (4.89). In this alternative result, the multiplicative factor preceding the exponential is smaller than (4.91) but the estimated decay rate is also smaller than (4.90) and depends on the quantity $H(0)$. To derive this alternative result, we first observe that for $b>a>1$,

$$
\ln \left(\frac{b-1}{a-1}\right)=\int_{a}^{b} \frac{d s}{s-1} \geq \frac{b-a}{b-1} .
$$

Setting

$$
b=\left\{H(0)+d^{2}\right\}^{1 / 2} / d, \quad a=\left\{H(z)+d^{2}\right\}^{1 / 2} / d
$$

in (4.92), we obtain

$$
\left\{H(0)+d^{2}\right\}^{1 / 2}-\left\{H(z)+d^{2}\right\}^{1 / 2} \leq\left\{\left[H(0)+d^{2}\right]^{1 / 2}-d\right\} \ln \left\{\frac{\left[H(0)+d^{2}\right]^{1 / 2}-d}{\left[H(z)+d^{2}\right]^{1 / 2}-d}\right\} .
$$

Using (4.94) in (4.86) leads to

$$
\ln \left\{\frac{\left[H(0)+d^{2}\right]^{1 / 2}-d}{\left[H(z)+d^{2}\right]^{1 / 2}-d}\right\} \geq \frac{z}{2 B^{1 / 2}\left[H(0)+d^{2}\right]^{1 / 2}},
$$

which we write as

$$
\left[H(z)+d^{2}\right]^{1 / 2}-d \leq\left\{\left[H(0)+d^{2}\right]^{1 / 2}-d\right\} e^{-z / 2 B^{1 / 2}\left[H(0)+d^{2}\right]^{1 / 2}} .
$$

Multiplying both sides of $(4.96)$ by $\left[H(z)+d^{2}\right]^{1 / 2}+d$ and recalling that $H(z) \leq$ $H(0)$, we deduce that

$$
H(z) \leq H(0) e^{-z / 2 B^{1 / 2}\left[H(0)+d^{2}\right]^{1 / 2}} .
$$

Since $H(z) \geq E(z)$, we thus obtain from (4.97) the result

$$
E(z) \leq H(0) e^{-z / 2 B^{1 / 2}\left[H(0)+d^{2}\right]^{1 / 2}}, \quad 0 \leq z \leq l .
$$


On comparing (4.98) with (4.89), we see that the multiplicative factor preceding the exponential in the former is smaller but the estimated decay rate in (4.98) is also smaller than that of (4.89) and depends on $H(0)$. For these reasons, we shall henceforth confine our attention to the result (4.89).

It remains to consider the case when $K=0$ in (4.3). In this case we find from (4.64), (4.65) that

$$
C=2\left(\lambda_{1}\right)^{-1 / 2}(M / m)^{1 / 2}, \quad D=0 .
$$

Thus (4.63) can be written as

$$
E^{\prime}(z)+\left(\frac{4 \lambda_{2}}{\lambda_{1}}\right)^{1 / 2} \frac{(1-\sigma)}{2 C} \int_{z}^{l} E(s) d s \leq\left(\frac{4 \lambda_{2}}{\lambda_{1}}\right)^{1 / 2} E(z) .
$$

It is convenient to rewrite $(4.100)$ as

$$
E^{\prime}(z)+4 \kappa^{2} \int_{z}^{l} E(s) d s \leq 4 \kappa^{2} a E(z)
$$

where

$$
\begin{gathered}
4 \kappa^{2}=\left(\frac{4 \lambda_{2}}{\lambda_{1}}\right)^{1 / 2} \frac{(1-\sigma)}{2 C}=\frac{(1-\sigma) \pi^{2}}{2 h^{2}}\left(\frac{3}{2}\right)^{2}\left(\frac{m}{M}\right)^{1 / 2}, \\
a=\frac{2 C}{(1-\sigma)}=\frac{4 h(M / m)^{1 / 2}}{\pi(1-\sigma)} .
\end{gathered}
$$

To integrate $(4.101)$, it is convenient to introduce the notation

$$
G(z)=\int_{z}^{l} E(s) d s
$$

so that

$$
G^{\prime}(z)=-E(z), \quad G^{\prime \prime}(z)=-E^{\prime}(z) .
$$

Thus we write $(4.101)$ as

$$
G^{\prime \prime}-4 \kappa^{2} a G^{\prime}(z)-4 \kappa^{2} G(z) \equiv(d / d z-\hat{\alpha})(d / d z+\hat{\beta}) G \geq 0,
$$

where the positive constants $\hat{\alpha}$ and $\hat{\beta}$ are given by

$$
\begin{aligned}
& \hat{\alpha}=2 \kappa\left[\kappa a+\sqrt{\kappa^{2} a^{2}+1}\right], \\
& \hat{\beta}=2 \kappa\left[-\kappa a+\sqrt{\kappa^{2} a^{2}+1}\right] .
\end{aligned}
$$

Integrating (4.106) with respect to $z$ from $z=0$ to $z=l$ and noting that $G(l)=0$ and $G^{\prime}(l)=0$, we obtain

$$
G^{\prime}(z)+\beta G(z) \leq 0, \quad 0 \leq z \leq l .
$$

On rewriting $(4.106)$ in the form

$$
(d / d z+\hat{\beta})(d / d z-\hat{\alpha}) G \geq 0
$$


and integrating from 0 to $z$, we find that

$$
\begin{aligned}
-G^{\prime}(z)+\hat{\alpha} G(z) & \leq\left[-G^{\prime}(0)+\alpha G(0)\right] e^{-\hat{\beta} z} \\
& \leq(1+\hat{\alpha} / \hat{\beta})\left[-G^{\prime}(0)\right] e^{-\hat{\beta} z}, \quad 0 \leq z \leq l,
\end{aligned}
$$

where the last inequality in (4.111) has been obtained on using (4.109) at $z=0$. By virtue of $(4.104),(4.105)$ we may write $(4.111)$ as

$$
E(z)+\hat{\alpha} \int_{z}^{l} E(s) d s \leq(1+\hat{\alpha} / \hat{\beta}) E(0) e^{-\hat{\beta} z},
$$

which implies that

$$
E(z) \leq(1+\hat{\alpha} / \hat{\beta}) E(0) e^{-\hat{\beta} z}, \quad 0 \leq z \leq l,
$$

where $\hat{\alpha}$ and $\hat{\beta}$ are given by (4.107) and (4.108) respectively. The result (4.113) is equivalent to (4.7) where

$$
\begin{gathered}
\frac{K_{1}}{E(0)}=1+\frac{\hat{\alpha}}{\hat{\beta}}=1+\left\{\kappa a+\left(\kappa^{2} a^{2}+1\right)^{1 / 2}\right\}^{2} \\
=1+\left\{\frac{3(M / m)^{1 / 4}}{[2(1-\sigma)]^{1 / 2}}+\left[\frac{9(M / m)^{1 / 2}}{2(1-\sigma)}+1\right]^{1 / 2}\right\}^{2}, \\
2 k=\hat{\beta}=2 \kappa\left\{\left(\kappa^{2} a^{2}+1\right)^{1 / 2}-\kappa a\right\} \\
=\frac{\pi}{h}\left(\frac{3}{2}\right)\left(\frac{m}{M}\right)^{1 / 4}\left(\frac{1-\sigma}{2}\right)^{1 / 2}\left\{\left[\frac{9(M / m)^{1 / 2}}{2(1-\sigma)}+1\right]^{1 / 2}-\frac{3(M / m)^{1 / 4}}{[2(1-\sigma)]^{1 / 2}}\right\} .
\end{gathered}
$$

This completes the derivation of the decay estimate (4.7) when the constant $K$ in (4.3) is zero.

5. Discussion. It is instructive to examine the decay estimate for the particular case when

$$
\rho=1+q^{2} \quad\left(q^{2}=\phi_{, \alpha \beta} \phi_{, \alpha \beta}\right) .
$$

As we have pointed out at the beginning of Sec. 4, we may take

$$
m=1, \quad M=1, \quad K=1, \quad \delta=\frac{1}{2}, \quad \sigma=0
$$

in this case. Thus the constants $C$ and $D$ given by (4.64) and (4.65) respectively are

$$
\begin{gathered}
C=2^{1 / 2}\left(2^{1 / 2}+\tau\right) h / \pi, \\
D=2^{9 / 4}(\Omega)^{-1 / 2},
\end{gathered}
$$

where

$$
\Omega=\Omega(2)
$$


is the Sobolev constant appearing in the inequality (4.36). It is shown in Appendix A that an admissible choice for $\Omega(2)$ is

$$
\Omega(2)=\pi / h,
$$

so that $D$ in (5.4) may be written as

$$
D=2^{9 / 4}\left(\frac{h}{\pi}\right)^{1 / 2} .
$$

With $C$ given by (5.3), we find from (4.76), with $\delta=\frac{1}{2}, \sigma=0$, and $\lambda_{1}, \lambda_{2}$ given by $(4.30),(4.49)$ respectively, that

$$
\beta_{*}=\frac{1}{2}\left\{\left[\frac{49}{4}+\frac{2^{7 / 2}}{9\left(2^{1 / 2}+\tau\right)}\right]^{1 / 2}-\frac{7}{2}\right\} .
$$

It is also shown in Appendix A that an admissible choice for the constant $\omega(2)$ in the Sobolev inequality (4.54) when $\delta=\frac{1}{2}$ is

$$
\omega(2)=\pi / h^{3 / 2} \text {. }
$$

Thus from (4.80) we find that

$$
A=\frac{2 h}{\beta_{*} \pi}\left\{\frac{2}{9}+\frac{3}{16}\left(\frac{\pi}{4}\right)^{1 / 3}\left[\beta_{*} 2^{1 / 2}\left(2^{1 / 2}+\tau\right)\right]^{-2 / 3}\right\} .
$$

Similarly, from (4.81) we get

$$
B=2^{7} \beta_{*}\left(2^{1 / 2}+\tau\right)^{-2} .
$$

The estimated decay rate given by (4.90) can be written explicitly as

$$
2 k=\frac{1}{A}=\frac{\pi}{h} \frac{\beta_{*}}{2}\left\{\frac{2}{9}+\frac{3}{16}\left(\frac{\pi}{4}\right)^{1 / 3}\left[\beta_{*} 2^{1 / 2}\left(2^{1 / 2}+\tau\right)\right]^{-2 / 3}\right\}^{-1},
$$

where $\beta_{*}$ is given in (5.8). Thus, for the material (5.1), we have the decay estimate (4.7) with decay rate $k$ given by (5.12).

It can be readily verified from $(5.12),(5.8)$ that $k=k^{*}(\tau) / h$ is a decreasing function of $\tau$ as $\tau$ increases, and that $k(\tau) \rightarrow 0$ as $\tau \rightarrow \propto$. Thus the results predict a progressively slower decay of end effects with increasing load level for the material (5.1). An analogous result was obtained, in the context of antiplane shear deformations, in $[1,26]$ for power-law materials which are hardening in shear. For the special case of self-equilibrated end loading where $\tau=0$, we obtain from (4.2) that $\psi=\phi$. In this case, we find from $(5.12),(5.8)$ that the estimated decay rate is

$$
k=0.0535 / h \text {. }
$$

For general materials for which (4.3) and (4.5) hold, the estimated decay rate is

$$
k=1 / 2 A,
$$


where $A$ is given by (4.80). The expression on the right-hand side of (4.80) involves the constant $C$, given by (4.64), and $\beta_{*}$ given by (4.76). It can be shown that as $\tau \rightarrow \infty, k(\tau) \rightarrow 0$, again predicting no attenuation of end effects in this limit.

It should be noted that, while the estimated decay rate $k$ given by $(5.14)$ for general materials depends on the load level $\tau$, in the special case when $K=0$ in (4.3) so that $\rho$ is a bounded function of its arguments, $k$ is independent of $\tau$. This can be seen immediately from the explicit result for $k$ given in (4.115).

6. Some extensions. We present here a sketch of some possible extensions. The first treats the same physical problem but with an assumption on $\rho$ which is different from (4.3), and the second retains assumption (4.3), but considers instead of the tension problem the case in which the rectangle is subjected to a uniform shear on the long sides and on the far end. We do not investigate the shear problem under the new assumptions on $\rho$ but we anticipate no difficulty in carrying through the required analysis in this case. Finally, an extension to some other boundary conditions is outlined.

We note that the analysis of the previous sections could not be applied if $\rho$ were, for instance, of the form

$$
\rho=\left(1+q^{2}\right)^{-\alpha}, \quad 0<\alpha<\frac{1}{2},
$$

since for such a $\rho$ we could not determine a positive constant $m$ in assumption (4.3). In $[28,29]$ the authors showed that similar decay results could be obtained in related second-order problems regardless of whether a hypothesis of type (4.3) is imposed on $\rho$ or imposed on $\rho^{-1}$. Following this idea we now show that, for the fourth-order problem of concern here, if instead of (4.3) we impose the hypothesis

$$
0<m_{1} \leq \rho^{-1} \leq M_{1}+K_{1}\left(\rho q^{2}\right)^{1-\delta}, \quad 0<\delta<1\left(m_{1}>0, M_{1}>0, K_{1} \geq 0\right),
$$

it is possible to again arrive at an inequality of the form (4.68) with, however, somewhat different constants. Then following the subsequent steps in Sec. 4 we would obtain the desired exponential decay. Before proceeding we remark that (6.1) satisfies (6.2) with $m_{1}=M_{1}=K_{1}=1$ and $\delta=(1-2 \alpha) /(1-\alpha)$.

The proof of our result under hypothesis (6.2) follows precisely the same lines as the proof using assumption (4.3) up to and including (4.20), but now instead of (4.21) we use the bound

$$
\begin{aligned}
J_{2} & \leq \frac{1}{m_{1}^{1 / 2}}\left\{\int_{R_{z}} \rho \psi_{, 1 \alpha} \psi_{, 1 \alpha} d A\right\}^{1 / 2}\left\{\int_{R_{z}} \psi_{, \alpha} \psi_{, \alpha} d A\right\}^{1 / 2} \\
& \leq \frac{1}{m_{1}^{1 / 2}}\{E(z)\}^{1 / 2}\left\{\int_{R_{z}} \psi_{, \alpha} \psi_{, \alpha} d A\right\}^{1 / 2} .
\end{aligned}
$$

We have used the left-hand inequality of (6.2) and the fact that $\phi_{, 1 \alpha}=\psi_{, 1 \alpha}$, as 
follows from (4.2). Now

$$
\begin{aligned}
\int_{R_{z}} \psi_{, \alpha} \psi_{, \alpha} d A=-\int_{L_{z}} \psi \psi \psi_{, 1} d x_{2}-\int_{R_{z}} \psi \psi_{, 2 \alpha} d A \\
\quad \leq\left\{\int_{L_{z}} \psi^{2} d x_{2} \int_{L_{z}} \psi_{, 1}^{2} d x_{2}\right\}^{1 / 2}+\left\{\int_{R_{z}} \rho^{-1} \psi^{2} d A \int_{R_{z}} \rho \psi_{, \alpha \alpha} \psi_{, \beta \beta} d A\right\}^{1 / 2} \\
\quad \leq\left\{p^{-1} \int_{R_{z}} \psi_{, \alpha} \psi_{, \alpha} d A \int_{L_{z}} \psi_{, 1}^{2} d x_{2}\right\}^{1 / 2}+\sqrt{2}\left\{\left(\int_{R_{z}} \rho^{-1} \psi^{2} d A\right) E(z)\right\}^{1 / 2}
\end{aligned}
$$

where $p(\geq \pi / h)$ is the first (Stekloff) eigenvalue for the problem (cf. [26, Appendix B])

$$
\begin{gathered}
\Delta \psi=0 \quad \text { on } R_{z}, \quad \psi\left(x_{1}, 0\right)=\psi\left(x_{1}, h\right)=0, \quad z<x_{1} \leq l, \\
\psi_{, 1}\left(z, x_{2}\right)+p \psi\left(z, x_{2}\right)=0, \quad 0 \leq x_{2} \leq h .
\end{gathered}
$$

To obtain the last inequality in (6.4), we have also used $\psi_{, \alpha \beta} \psi_{, \alpha \beta} \geq \psi_{, \alpha \alpha} \psi_{, \beta \beta} / 2$. To complete the bound for $\int_{R_{z}} \psi_{, \alpha} \psi_{, \alpha} d A$ we thus need to bound the two quantities $\int_{L_{z}} \psi_{, 1}^{2} d x_{2}$ and $\int_{R_{z}} \rho^{-1} \psi^{2} d A$. Proceeding as in [28], using a change of variable in the cross-section, we have

$$
\int_{L_{z}} \psi_{, 1}^{2} d x_{2} \leq \frac{B^{2}}{\pi^{2}} \int_{L_{z}} \rho \psi_{, 1 \beta} \psi_{, 1 \beta} d x_{2} \leq \frac{B^{2}}{\pi^{2}}\left(-E^{\prime}(z)\right),
$$

where

$$
B(z)=\int_{L_{z}} \rho^{-1} d x_{2}
$$

Thus, on using (6.2) we find

$$
\begin{aligned}
B & \leq \int_{L_{z}}\left[M_{1}+K_{1}\left(\rho\left(q^{2}\right) q^{2}\right)^{1-\delta}\right] d x_{2} \\
& \leq \int_{L_{z}}\left\{M_{1}+2^{1-\delta} K_{1}\left[\rho\left(q^{2}\right) \psi_{, \alpha \beta} \psi_{, \alpha \beta}\right]^{1-\delta}+\left(2 \tau^{2}\right)^{1-\delta} K_{1} \rho^{1-\delta}\right\} d x_{2} \\
& \leq\left[M_{1}+\left(2 \tau^{2}\right)^{1-\delta} K_{1} m_{1}^{-(1-\delta)}\right] h+2^{1-\delta} K_{1} h^{\delta}\left[-E^{\prime}(z)\right]^{1-\delta},
\end{aligned}
$$

where the second inequality has been obtained using (4.24) and the final inequality follows on using Hölder's inequality. Combining (6.6) and (6.7) we find

$$
\left\{\int_{L_{z}} \psi_{, 1}^{2} d x_{2}\right\}^{1 / 2} \leq \frac{1}{\pi}\left\{A_{1}\left[-E^{\prime}(z)\right]^{1 / 2}+A_{2}\left[-E^{\prime}(z)\right]^{(3-\delta) / 2}\right\}
$$

with the obvious definitions of the constants $A_{1}$ and $A_{2}$. 
To bound $\int_{R_{z}} \rho^{-1} \psi^{2} d A$ we use (6.2) to write

$$
\begin{aligned}
\int_{R_{z}} \rho^{-1} \psi^{2} d A \leq & \int_{R_{z}}\left[M_{1}+K_{1}\left(\rho q^{2}\right)^{1-\delta}\right] \psi^{2} d A \\
\leq & \int_{R_{z}}\left[M_{1}+2^{1-\delta} K_{1}\left(\rho \psi_{, \alpha \beta} \psi_{, \alpha \beta}\right)^{1-\delta}+\left(2 \tau^{2}\right)^{1-\delta} K_{1} \rho^{1-\delta}\right] \psi^{2} d A \\
\leq & {\left[M_{1}+\left(2 \tau^{2}\right)^{1-\delta} K_{1}\left(m_{1}\right)^{-(1-\delta)}\right] h^{2} \pi^{-2} \int_{R_{z}} \psi_{, \alpha} \psi_{, \alpha} d A } \\
& +2^{1-\delta} K_{1}[E(z)]^{1-\delta}\left[\int_{R_{z}} \psi^{2 / \delta} d A\right]^{\delta} \\
\leq & \left\{A_{3}+2^{1-\delta} K_{1} \Omega^{-1}(1 / \delta)[E(z)]^{1-\delta}\right\} \int_{R_{z}} \psi_{, \alpha} \psi_{, \alpha} d A
\end{aligned}
$$

where the definition of $A_{3}$ is clear. We have used (4.24), Hölder's inequality, a version of (4.29), and the Sobolev inequality (4.36) with Sobolev constant $\Omega(1 / \delta)$.

We now insert (6.9) and (6.8) into (6.4) and solve to obtain

$$
\begin{aligned}
\left\{\int_{R_{z}} \psi_{, \alpha} \psi_{, \alpha} d A\right\}^{1 / 2} \leq & {\left[B_{1}+B_{2} \tau^{2(1-\delta)}\right]\left[-E^{\prime}(z)\right]^{1 / 2}+B_{3}\left[-E^{\prime}(z)\right]^{(3-2 \delta) / 2} } \\
& +\left[B_{4}+B_{5} \tau^{1-\delta}\right][E(z)]^{1 / 2}+B_{6}[E(z)]^{(2-\delta) / 2}
\end{aligned}
$$

for computable $B_{i}$. Note that the $B_{i}$ are independent of $\tau$. Thus (6.10) and (6.3) provide a bound for $J_{2}$ in terms of $E(z)$ and $E^{\prime}(z)$.

To bound the $J_{1}$ in $(4.20)$ we note that

$$
\begin{aligned}
J_{1} & \leq m^{-1 / 2}\left[-E^{\prime}(z)\right]^{1 / 2}\left(\int_{L_{z}} \psi^{2} d x_{2}\right)^{1 / 2} \\
& \leq\left(m_{1} p\right)^{-1 / 2}\left[-E^{\prime}(z)\right]^{1 / 2}\left(\int_{R_{z}} \psi_{, \alpha} \psi_{.,} d A\right)^{1 / 2},
\end{aligned}
$$

where $p \geq \pi / h$ is again the first Stekloff eigenvalue for the rectangle. Using (6.10) we obtain the desired bound for $J_{1}$.

We next insert the bounds for $J_{1}$ and $2 J_{2}$ into (4.20) and after simplification obtain

$$
\begin{aligned}
&(1-\sigma) \int_{z}^{l} E(s) d s \leq\{\left.m_{1}^{-1 / 2}[E(z)]^{1 / 2}+\left(m_{1} p\right)^{-1 / 2}\left[-E^{\prime}(z)\right]^{1 / 2}\right\} \\
& \cdot\left\{\left[B_{1}+B_{2} \tau^{2(1-\delta)}\right]\left[-E^{\prime}(z)\right]^{1 / 2}+B_{3}\left[-E^{\prime}(z)\right]^{(3-2 \delta) / 2}\right. \\
&\left.+\left[B_{4}+B_{5} \tau^{(1-\delta)}\right] E^{1 / 2}(z)+B_{6}[E(z)]^{(2-\delta) / 2}\right\} .
\end{aligned}
$$

If we now make use of arguments analogous to (4.67) (for different exponents) we can find computable constants $C_{i}>0$ such that

$$
\begin{aligned}
\left(\frac{1-\sigma}{2}\right) \int_{z}^{l} E(s) d s \leq & {\left[C_{1}+C_{2} \tau^{2(1-\delta)}+C_{3} \tau^{1-\delta}\right]\left\{E(z)+C_{4}\left[-E^{\prime}(z)\right]\right\} } \\
& +C_{4} E(z)^{2}+C_{5}\left[-E^{\prime}(z)\right]^{2},
\end{aligned}
$$


which leads directly to an inequality of the form (4.68).

From the way in which $\tau$ comes into the inequality (6.13) it can be seen that the decay constant $k=k^{*}(\tau) / h$ in the final estimate (4.7) is again a decreasing function of $\tau$ as $\tau$ increases and that $k(\tau) \rightarrow 0$ as $\tau \rightarrow \infty$.

The second extension we consider is that of a shear loading problem rather than the tensile load problem formulated in Secs. 2 and 3. Thus the boundary conditions (2.1)-(2.3) are replaced by

$$
\begin{array}{cll}
\tau_{12}\left(x_{1}, 0\right)=-\tau, \quad \tau_{22}\left(x_{1}, 0\right)=0, & \tau_{12}\left(x_{1}, h\right)=-\tau, & \tau_{22}\left(x_{1}, h\right)=0, \\
& 0<x_{1}<l, \\
& & \\
\tau_{11}\left(l, x_{2}\right)=0, & \tau_{12}\left(l, x_{2}\right)=-\tau, & 0 \leq x_{2} \leq h, \\
\tau_{11}\left(0, x_{2}\right)=f\left(x_{2}\right), & \tau_{12}\left(0, x_{2}\right)=g\left(x_{2}\right), & 0 \leq x_{2} \leq h,
\end{array}
$$

where the given constant $\tau$ is nonnegative. Here $f$ and $g$ are prescribed functions, continuous on $[0, h]$ and satisfy

$$
\begin{gathered}
g(0)=g(h)=-\tau, \\
\int_{0}^{h} f\left(x_{2}\right) d x_{2}=0, \quad \int_{0}^{h} x_{2} f\left(x_{2}\right) d x_{2}=0, \\
\int_{0}^{h} g\left(x_{2}\right) d x_{2}=-\tau h .
\end{gathered}
$$

If $g\left(x_{2}\right)$ were constant on $[0, h]$ (and thus by (6.19), $g=-\tau$ ), and $f \equiv 0$ on $[0, h]$, then the state of uniform shear

$$
\tau_{11}=0, \quad \tau_{22}=0, \quad \tau_{12}=-\tau \quad \text { on } R
$$

would again be a possible equilibrium solution, with corresponding Airy stress function

$$
\phi=\tau x_{1} x_{2} .
$$

Thus, when $g \neq$ constant, we are interested in the spatial evolution of the stresses to the uniform state $(6.20)$.

On integrating the boundary conditions $(6.14)-(6.16)$ twice, we arrive at the final boundary-value problem for $\phi$ analogous to that of Sec. 3. Thus we are concerned with solutions of (3.1) subject to the boundary conditions

$$
\begin{aligned}
& \phi\left(x_{1}, 0\right)=0, \quad \phi_{.2}\left(x_{1}, 0\right)=\tau x_{1}, \quad 0<x_{1}<l, \\
& \phi\left(x_{1}, h\right)=\tau h x_{1}, \quad \phi_{, 2}\left(x_{1}, h\right)=\tau x_{1}, \quad 0<x_{1}<l, \\
& \phi\left(l, x_{2}\right)=\tau l x_{2}, \quad \phi_{.1}\left(l, x_{2}\right)=\tau x_{2}, \quad 0 \leq x_{2} \leq h, \\
& \phi\left(0, x_{2}\right)=F\left(x_{2}\right), \quad \phi_{, 1}\left(0, x_{2}\right)=G\left(x_{2}\right), \quad 0 \leq x_{2} \leq h .
\end{aligned}
$$

Here the functions $F$ and $G$ are again given by (2.29) in terms of the $f$ and $g$ of (6.16) and satisfy

$$
\begin{aligned}
& F(0)=0, \quad F(h)=0, \quad F^{\prime}(0)=0, \quad F^{\prime}(h)=0, \\
& G(0)=0, \quad G(h)=\tau h .
\end{aligned}
$$


As in Sec. 4, we now define a quadratic functional $E(z)$ by $(4.1)$, where $\psi$ is defined by

$$
\psi\left(x_{1}, x_{2}\right)=\phi\left(x_{1}, x_{2}\right)-v\left(x_{1}, x_{2}\right), \quad v \equiv \tau x_{1} x_{2},
$$

and seek to establish a decay estimate of the form (4.7). We assume that $\rho$ satisfies the hypotheses (4.3), (4.5). It turns out that we also require an additional assumption of the form (4.5), namely that for a fixed value of the argument $s$ and for arbitrary values of $t$

$$
\left|\rho\left(s^{2}\right)-\rho\left(t^{2}\right)\right||s+t| \leq c\left[\rho\left(s^{2}\right)+\rho\left(t^{2}\right)\right]|s-t|
$$

for some nonnegative constant $c$. We note that when $\rho=1+q^{2}$, we may choose $c=2$ in (6.29).

An estimate of the form (4.7) for the present problem again follows from an integro-differential inequality of the form (4.63). To establish the differential inequality, we find on using the divergence theorem and (6.22)-(6.24), that (4.11) still holds. As in proceeding from (4.11) to (4.17), we find here that

$$
\begin{aligned}
E(z)(1-\sigma) / 2 \leq & -\int_{L_{z}}\left[\rho\left(q^{2}\right) \phi_{, 1 \alpha}-\rho\left(\tau^{2}\right) v_{, 1 \alpha}\right] \psi_{, \alpha} d x_{2} \\
& +\int_{L_{z}}\left[\rho\left(q^{2}\right) \phi_{, 1 \beta}-\rho\left(\tau^{2}\right) v_{, 1 \beta}\right]_{, \beta} \psi d x_{2} .
\end{aligned}
$$

The difference between (6.30) and (4.17) is due to the fact that here $v=v\left(x_{1}, x_{2}\right)$ (see (6.28)) while in Sec. 4, $v=v\left(x_{2}\right)$ (see (4.13)). An integration of (6.30) now leads to

$$
\begin{aligned}
\frac{(1-\sigma)}{2} \int_{z}^{l} E(s) d s \leq & -\int_{L_{z}}\left[\rho\left(q^{2}\right) \phi_{, 11}-\rho\left(\tau^{2}\right) v_{, 11}\right] \psi d x_{2} \\
& -2 \int_{R_{z}}\left[\rho\left(q^{2}\right) \phi_{, 1 \alpha}-\rho\left(\tau^{2}\right) v_{, 1 \alpha}\right] \psi_{, \alpha} d A,
\end{aligned}
$$

where the divergence theorem and the boundary conditions (6.22)-(6.24) have also been used. The right-hand side of $(6.31)$ is now written as

$$
\begin{aligned}
& -\int_{L_{z}} \rho\left(q^{2}\right) \psi_{, 11} \psi d x_{2}-\int_{R_{z}}\left[\rho\left(q^{2}\right)-\rho\left(\tau^{2}\right)\right]\left[\phi_{, 1 \alpha}+v_{, 1 \alpha}\right] \psi_{, \alpha} d A \\
& -\quad-\int_{R_{z}}\left[\rho\left(q^{2}\right)+\rho\left(\tau^{2}\right)\right]\left[\phi_{, 1 \alpha}-v_{, 1 \alpha}\right] \psi_{, \alpha} d A,
\end{aligned}
$$

and thus we find

$$
\begin{aligned}
\frac{(1-\sigma)}{2} \int_{z}^{l} E(s) d s \leq & -\int_{L_{z}} \rho\left(q^{2}\right) \psi_{, 11} \psi d x_{2}+\int_{R_{z}}\left|\rho\left(q^{2}\right)-\rho\left(\tau^{2}\right)\|q+\tau\| \psi_{, \alpha}\right| d A \\
& +\int_{R_{z}}\left[\rho\left(q^{2}\right)+\rho\left(\tau^{2}\right)\right] \psi_{, 1 \alpha} \psi_{, \alpha} d A
\end{aligned}
$$


which leads to

$$
\begin{aligned}
\frac{(1-\sigma)}{2} \int_{z}^{l} E(s) d s \leq & -\int_{L_{z}} \rho\left(q^{2}\right) \psi_{, 11} \psi d x_{2} \\
& +c \int_{R_{z}}\left[\rho\left(q^{2}\right)+\rho\left(\tau^{2}\right)\right]\left[\psi_{, \alpha \beta} \psi_{, \alpha \beta}\right]^{1 / 2}\left[\psi_{, \gamma} \psi_{, \gamma}\right]^{1 / 2} d A \\
& +\int_{R_{z}}\left[\rho\left(q^{2}\right)+\rho\left(\tau^{2}\right)\right]\left[\psi_{, \alpha \beta} \psi_{, \alpha \beta}\right]^{1 / 2}\left[\psi_{, \gamma} \psi_{, \gamma}\right]^{1 / 2} d A,
\end{aligned}
$$

where (6.29) and (4.15) have been used to obtain the final inequality in (6.34). Thus we deduce from (6.34) that

$$
\frac{(1-\sigma)}{2} \int_{z}^{l} E(s) d s \leq J_{1}+(c+1) T,
$$

where

$$
T \equiv \int_{R_{z}}\left[\rho\left(q^{2}\right)+\rho\left(\tau^{2}\right)\right]\left(\psi_{, \alpha \beta} \psi_{, \alpha \beta}\right)^{1 / 2}\left(\psi_{, \gamma} \psi_{, \gamma}\right)^{1 / 2} d A,
$$

and $J_{1}$ is exactly as defined in (4.20). Comparing (4.20) and (6.35), we see that $(c+1) T$ in (6.35) plays the role of $2 J_{2}$ in (4.20). For $J_{1}$ we use the bound (4.62) and so to complete the derivation of an inequality of the form (4.63) it remains to obtain an upper bound for $T$. The structure of $T$ is similar to that of $J_{2}$ defined in (4.20). Proceeding as in Sec. 4, it can be shown that $T$ satisfies an inequality of the form (4.43), namely

$$
T \leq C_{1} E(z)+C_{2}[E(z)]^{(3-\delta) / 2}
$$

for some constants $C_{1}$ and $C_{2}$. Thus, on using (4.62) and (6.37) in (6.35) one arrives at the desired inequality of the form (4.63), from which one can proceed formally as in Sec. 4 to establish a final decay estimate of the form (4.7) for the shear problem.

We have also developed the method to handle some other boundary conditions. For instance, we may consider the case in which the long sides of the rectangle are subjected to a uniform tensile traction $\tau \geq 0$, and the far end is traction free. The mathematical problem is quite similar to that of the shear problem. The solution corresponding to a traction-free near end is given by the particular solution

$$
\phi=\tau x_{1}^{2} / 2
$$

of (2.14). For nonzero (but necessarily self-equilibrated end tractions) we are again concerned with the decay of $E(z)$, where $\psi=\phi-v, v=\tau x_{1}^{2} / 2$, and $\phi$ satisfies (2.14), while $\psi$ satisfies

$$
\begin{gathered}
\psi\left(x_{1}, 0\right)=\psi_{, 2}\left(x_{1}, 0\right)=\psi\left(x_{1}, h\right)=\psi_{.2}\left(x_{1}, h\right)=0, \quad 0 \leq x_{1} \leq l, \\
\psi\left(l, x_{2}\right)=\psi ._{1}\left(l, x_{2}\right)=0, \quad 0 \leq x_{2} \leq h .
\end{gathered}
$$

The derivation of the bound for $\int_{z}^{l} E(s) d s$ in this case proceeds as in the shear problem as far as $(6.31)$. However, now $v_{, 11} \neq 0$. If we handle the line integral in 
(6.31) in the same way in which we treated the area integral for the shear problem we arrive at $(6.35)$, but now the term $J_{1}$ is replaced by $\widetilde{J}_{1}$ given by

$$
\widetilde{J}_{1}=\frac{1}{2}(c+1) \int_{L_{z}}\left[\rho\left(q^{2}\right)+\rho\left(\tau^{2}\right)\right]\left[\psi_{, \alpha \beta} \psi_{, \alpha \beta}\right]^{1 / 2}|\psi| d x_{2} .
$$

The decay estimate now follows as before.

More generally if $v\left(x_{1}, x_{2}\right)$ is any solution of $(2.14)$ and $\phi\left(x_{1}, x_{2}\right)$ is any other solution of (2.14) satisfying the same Dirichlet boundary conditions as $v$ on the long sides and far end of the rectangle, then the arguments of this paper may be modified to show that $\phi-v$ decays exponentially in energy norm as $x_{1}$ increases. Letting

$$
\psi=\phi-v
$$

we are faced with two cases. In the first case if $v=v\left(x_{2}\right)$ then the results of Sec. 4 carry through directly with the $\tau$ which appears in the definition of the constants $C$ and $D$ replaced by $\max _{0 \leq x_{2} \leq h}\left|v^{\prime \prime}\right|$. It is dlso understood that in the definition (4.1) of $E, \rho\left(\tau^{2}\right)$ is really $\rho\left(\left(v^{\prime \prime}\right)^{2}\right)$. Note that the hypothesis (6.29) is not required. On the other hand if $v=v\left(x_{1}, x_{2}\right)$, then following the steps already described in this section one arrives at

$$
\frac{(1-\sigma)}{2} \int_{z}^{l} E(s) d s \leq(c+1)\left[\widetilde{J}_{1}+T\right],
$$

where $\widetilde{J}_{1}$ is given by $(6.40)$ and $T$ by (6.36) (with $\rho\left(\tau^{2}\right)$ given by $\rho\left(v_{{ }_{\alpha \beta}} v_{, \alpha \beta}\right)$ and the $\tau$ which appears in the resulting constants replaced by $\left.\max _{R}\left[v_{, \alpha \beta} v_{, \alpha \beta}\right]^{1 / 2}\right)$. Again the desired decay estimate then follows from the arguments of Sec. 4.

Appendix A. The Sobolev inequalities (4.36) and (4.54). Sobolev inequalities of the form (4.36) are well known for very general domains provided that $\psi$ has compact support. In our problem $\psi$ does not vanish on $L_{z}$. For this reason and since we wish to obtain an explicit bound for the constant $\Omega(1 / \delta)$ appearing in (4.36), it is convenient to appeal to a direct proof of (4.36) for the rectangular domain $R_{z}$ of concern here. Similar issues were considered in [41]. In what follows for simplicity we present a derivation of the scalar version of (4.36). The same arguments for vector functions would yield (4.36) with the same value for the Sobolev constant.

For sufficiently smooth functions $v\left(x_{1}, x_{2}\right)$ that vanish on the lateral sides and on the end $z=l$ of $R_{z}$, it is shown in Appendix A of [41] (see Eqs. (A.16) of [41]) that

$$
\int_{R_{z}}|v|^{2 / \delta} d A \leq \frac{1}{4 \delta^{2}}\left(\int_{R_{z}}|v|^{2(1-\delta) / \delta} d A\right)\left(\int_{R_{z}}{ }{ }{ }_{, \alpha}{ }{ }{ }_{, \alpha} d A\right)
$$

for $0<\delta \leq 1$. Suppose first that $\delta \leq \frac{1}{2}$. The first integral on the right in (A.1) may be written as

$$
\begin{aligned}
& \int_{R_{z}}\left(|v|^{2 / \delta}\right)^{(1-2 \delta) /(1-\delta)}\left(v^{2}\right)^{\delta /(1-\delta)} d A \\
& \quad \leq\left(\int_{R_{z}}|v|^{2 / \delta} d A\right)^{(1-2 \delta) /(1-\delta)}\left(\int_{R_{z}} v^{2} d A\right)^{\delta /(1-\delta)},
\end{aligned}
$$


where Hölder's inequality has been used to obtain (A.3). Thus, for $0<\delta \leq 1 / 2$, we find from (A.1) and (A.3) that

$$
\left(\int_{R_{z}}|v|^{2 / \delta} d A\right)^{\delta /(1-\delta)} \leq \frac{1}{4 \delta^{2}}\left(\int_{R_{z}} v^{2} d A\right)^{\delta /(1-\delta)}\left(\int_{R_{z}} v_{, \alpha} v_{, \alpha} d A\right) .
$$

Since $v\left(x_{1}, x_{2}\right)=0$ at the end points of $L_{z}$, we have, by Wirtinger's inequality,

$$
\int_{L_{z}} v_{, 2}^{2} d x_{2} \geq \frac{\pi^{2}}{h^{2}} \int_{L_{z}} v^{2} d x_{2}, \quad 0 \leq z \leq l
$$

so that

$$
\int_{R_{z}} v^{2} d A \leq \frac{h^{2}}{\pi^{2}} \int_{R_{z}} v_{, \alpha} v_{, \alpha} d A .
$$

Using (A.6) in (A.4) we find that

$$
\int_{R_{z}}|v|^{2 / \delta} d A \leq\left(\frac{1}{4 \delta^{2}}\right)^{(1-\delta) / \delta} \frac{h^{2}}{\pi^{2}}\left(\int_{R_{z}} v_{, \alpha} v_{, \alpha} d A\right)^{1 / \delta}, \quad 0<\delta \leq \frac{1}{2},
$$

or equivalently

$$
\left(\int_{R_{z}}|v|^{2 / \delta} d A\right)^{\delta} \leq\left(\frac{1}{4 \delta^{2}}\right)^{1-\delta}\left(\frac{h}{\pi}\right)^{2 \delta} \int_{R_{z}} v_{, \alpha} v_{, \alpha} d A, \quad 0<\delta \leq \frac{1}{2} .
$$

Thus we obtain the Sobolev inequality (4.36), with Sobolev constant $\Omega(1 / \delta)$ given by

$$
\Omega(1 / \delta)=\left(4 \delta^{2}\right)^{1-\delta}(\pi / h)^{2 \delta}, \quad 0<\delta \leq \frac{1}{2} .
$$

In particular, for $\delta=\frac{1}{2}$, we have

$$
\Omega(2)=\pi / h \text {. }
$$

When $\frac{1}{2}<\delta<1$, we use Schwarz's inequality to obtain

$$
\int_{R_{z}}|v|^{2 / \delta} d A \leq\left(\int_{R_{z}} v^{2} d A\right)^{1 / 2}\left(\int_{R_{z}}|v|^{2(2-\delta) / \delta} d A\right)^{1 / 2} .
$$

Employing (A.7) with $\delta$ replaced by $\delta /(2-\delta)$ to bound the second integral on the right in (A.11), we find from (A.11) that

$$
\begin{aligned}
\int_{R_{z}}|v|^{2 / \delta} d A & \leq\left(\int_{R_{z}} v^{2} d A\right)^{1 / 2}\left[\left\{\frac{(2-\delta)^{2}}{4 \delta^{2}}\right\}^{2(1-\delta) / \delta} \frac{h^{2}}{\pi^{2}}\left(\int_{R_{z}} v_{, \alpha} v_{, \alpha} d A\right)^{(2-\delta) / \delta}\right]^{1 / 2} \\
& \leq\left\{\frac{(2-\delta)^{2}}{4 \delta^{2}}\right\}^{(1-\delta) / \delta} \frac{h^{2}}{\pi^{2}}\left(\int_{R_{z}} v_{, \alpha} v_{, \alpha} d A\right)^{1 / \delta}
\end{aligned}
$$

where (A.6) has been used to obtain the last inequality in (A.12). The inequality (A.12) may be written as

$$
\left(\int_{R_{z}}|v|^{2 / \delta} d A\right)^{\delta} \leq\left(\frac{2-\delta}{2 \delta}\right)^{2(1-\delta)}\left(\frac{h}{\pi}\right)^{2 \delta} \int_{R_{z}} v_{, \alpha} v_{, \alpha} d A, \quad \frac{1}{2}<\delta<1 .
$$


Thus we obtain the Sobolev inequality (4.36) with Sobolev constant $\Omega(1 / \delta)$ given by

$$
\Omega\left(\frac{1}{\delta}\right)=\left(\frac{2 \delta}{2-\delta}\right)^{2(1-\delta)}\left(\frac{\pi}{h}\right)^{2 \delta}, \quad \frac{1}{2}<\delta<1 .
$$

The one-dimensional Sobolev inequality (4.54) for functions $\psi$ which vanish at the end points of $L_{z}$ is also well known. The optimal Sobolev constant $\omega(1 / \delta)$ appearing in (4.54) can be characterized as an eigenvalue using a variational approach. For simplicity here, we merely record admissible values for $\omega(1 / \delta)$, which are not optimal, except in the trivial case when $\delta=1$. Thus, starting from the inequality

$$
|\psi|^{1 / \delta} \leq \frac{1}{2 \delta} \int_{0}^{h}|\psi|^{(1-\delta) / \delta}\left|\psi_{, 2}\right| d x_{2},
$$

and using Schwarz's inequality, it is readily shown that (4.54) holds with constants

$$
\begin{aligned}
& \omega\left(\frac{1}{\delta}\right)=\left(4 \delta^{2}\right)^{1-\delta} \pi^{2 \delta} / h^{1+\delta}, \quad 0<\delta \leq \frac{1}{2}, \\
& \omega\left(\frac{1}{\delta}\right)=\left(\frac{2 \delta}{2-\delta}\right)^{2(1--\delta)} \pi^{2 \delta} / h^{1+\delta}, \quad \frac{1}{2}<\delta \leq 1 .
\end{aligned}
$$

In particular, when $\delta=\frac{1}{2}$, we obtain from (A.16)

$$
\omega(2)=\pi / h^{3 / 2} \text {. }
$$

Appendix B. An upper bound for $K_{1}$. The decay estimate (4.7), or equivalently (4.89), has a multiplicative factor $K_{1}=K_{1}\left(E_{0}, m, M, K, \delta, \sigma\right)$, where $E_{0} \equiv E(0)$ is the total weighted energy contained in the rectangle $R \equiv R_{0}$. When the constant $K$ in (4.3) is zero, $K_{1} / E(0)$ is given explicitly by (4.114) in terms of $m, M$, and the function $\sigma$ appearing in (4.5) and so, in this case, $K_{1}$ is a multiple of $E(0)$. When $K \neq 0, K_{1}$ is given by (4.91). To obtain an upper bound for $K_{1}$ in this case, we require an upper bound for the quantity $H(0)$, which is given from (4.79) as

$$
H(0)=E(0)+\left(\beta_{*} C\right)^{-1} \int_{0}^{l} E(s) d s .
$$

Thus in both cases we seek upper bounds for $E(0)$ (as well as for $\int_{0}^{l} E(s) d s$ when $K \neq 0$ ) in terms of the geometry and boundary data. Bounds of this type for $E(0)$ for second-order problems have been discussed in our previous work [28] (see also [2]).

We begin with the inequality (4.19) evaluated at $z=0$, which reads

$$
\frac{(1-\sigma)}{2} \int_{0}^{l} E(s) d s \leq-\int_{L_{0}} \rho\left(q^{2}\right) \phi_{, 11}(F-v) d x_{2}-2 \int_{R} \rho\left(q^{2}\right) \phi_{, 1 \alpha} \psi_{, \alpha} d A \equiv I_{1}+I_{2},
$$

where we have used the fact that $\psi=\phi-v$ and the boundary condition (3.6), . Our first objective is to obtain an upper bound for $\int_{0}^{l} E(s) d s$ in terms of $E(0)$. Subsequently we will establish an upper bound for $E(0)$. 
From its definition in (B.2), we have

$$
\begin{aligned}
I_{2} & \leq 2 \int_{R} \rho\left(q^{2}\right)\left(\phi_{, 1 \beta} \phi_{, 1 \beta}\right)^{1 / 2}\left(\psi_{, \gamma} \psi_{, \gamma}\right)^{1 / 2} d A, \\
& =2 \int_{R} \rho\left(q^{2}\right)\left(\psi_{, 1 \beta} \psi_{, 1 \beta}\right)^{1 / 2}\left(\psi_{, \gamma} \psi_{, \gamma}\right)^{1 / 2} d A \equiv 2 I_{2}^{*} .
\end{aligned}
$$

On comparing (B.4) with (4.20), it is clear that the integral $I_{2}^{*}$ is identical to the integral $J_{2}=J_{2}(z)$ evaluated at $z=0$. Thus, from (4.43) evaluated at $z=0$ and (B.4) we obtain the upper bound

$$
\begin{aligned}
I_{2} \leq 2 I_{2}^{*} \leq & 2\left(\lambda_{1} m\right)^{-1 / 2}\left[M^{1 / 2}+2^{1-\delta} K^{1 / 2} \delta \tau^{1-\delta}\left\{2(1-\delta) \tau^{1-\delta} 2^{1-\delta} K^{1 / 2}\right\}^{(1-\delta) / \delta}\right] E(0) \\
& +4\left(2^{1-\delta}\right) K^{1 / 2}(\Omega m)^{-1 / 2} E(0)^{(3-\delta) / 2} \\
= & C E(0)+D E(0)^{(3-\delta) / 2}
\end{aligned}
$$

where $C$ and $D$ are defined in (4.64) and (4.65) respectively.

To obtain an upper bound for the integral $I_{1}$ defined in (B.2) we introduce the function

$$
w\left(x_{1}, x_{2}\right)=x_{1}(F-v)\left(e^{-a x_{1}}-e^{-a l}\right)^{2} /\left(1-e^{-a l}\right)^{2},
$$

where $a>0$ is an arbitrary constant. Assuming that the function $F\left(x_{2}\right)$ appearing in the boundary condition (3.6) is sufficiently smooth so that

$$
F\left(x_{2}\right)=v\left(x_{2}\right), \quad \frac{d F}{d x_{2}}=\frac{d v}{d x_{2}} \quad \text { at } x_{2}=0, h,
$$

it can be readily verified that $w$ as defined in (B.7) satisfies the boundary conditions

$$
\begin{gathered}
w=0 \quad \text { on } \partial R, \\
w_{, 2}=0 \quad \text { on } x_{2}=0, h\left(0 \leq x_{1} \leq l\right), \\
w_{, 1}=0 \quad \text { on } x_{1}=l, \quad w_{, 1}=F-v \quad \text { on } x_{1}=0\left(0 \leq x_{2} \leq h\right) .
\end{gathered}
$$

On using the divergence theorem and the boundary conditions (B.9)-(B.11), it is easily shown that $I_{1}$ can be written as

$$
I_{1}=\int_{R} \rho\left(q^{2}\right) \phi_{, \alpha \beta} w_{, \alpha \beta} d A=\int_{R} \rho\left(q^{2}\right)\left(\psi_{, \alpha \beta}+\tau \delta_{\kappa 2} \delta_{\beta 2}\right) w_{, \alpha \beta} d A,
$$

which yields by Schwarz's inequality

$$
I_{1} \leq\{E(0)\}^{1 / 2}\left\{\int_{R} \rho\left(q^{2}\right) w_{, \alpha \beta} w_{, \alpha \beta} d A\right\}^{1 / 2}+\tau \int_{R} \rho\left(q^{2}\right)\left|w_{, 22}\right| d A .
$$

We see then that to complete the bound for $I_{1}$ we require bounds for two integrals of the form $\int_{R} \rho\left(q^{2}\right) P^{2} d A$, where $P$ is a known quantity. We observe that

$$
\begin{aligned}
S & \equiv \int_{R} \rho\left(q^{2}\right) P^{2} d A \leq \int_{R} \rho^{1 / 2}\left[M+K\left(\rho q^{2}\right)^{1-\delta}\right]^{1 / 2} P^{2} d A \\
& \leq M^{1 / 2} \int_{r} \rho^{1 / 2} P^{2} d A+K^{1 / 2} \int_{R} \rho^{1-\delta / 2}\left(q^{2}\right)^{(1-\delta) / 2} P^{2} d A,
\end{aligned}
$$


where we have made use of assumption (4.3). Employing Schwarz's inequality and (4.24) we thus obtain

$$
\begin{aligned}
S \leq & M^{1 / 2} S^{1 / 2}\left\{\int_{R} P^{2} d A\right\}^{1 / 2}+2^{(1-\delta) / 2} K^{1 / 2} \int_{R} \rho^{1-\delta / 2}\left(\psi_{, \alpha \beta} \psi_{, \alpha \beta}\right)^{(1-\delta) / 2} P^{2} d A \\
& +\left(2 \tau^{2}\right)^{(1-\delta) / 2} K^{1 / 2} \int_{R} \rho^{1-\delta / 2} P^{2} d A .
\end{aligned}
$$

An application of (4.34) in the second and third terms with appropriate choices for $f, g, p$, and $q$ now leads to

$$
\begin{aligned}
S \leq & M^{1 / 2} S^{1 / 2}\left\{\int_{R} P^{2} d A\right\}^{1 / 2}+2^{(1-\delta) / 2} K^{1 / 2} E(0)^{(1-\delta) / 2}\left\{\int_{R} \rho^{1 /(1+\delta)} P^{4 /(1+\delta)} d A\right\} \\
& +\left(2 \tau^{2}\right)^{(1-\delta) / 2} K^{1 / 2} S^{1-\delta / 2}\left(\int_{R} P^{2} d A\right)^{\delta / 2}, \\
\leq & M^{1 / 2} S^{1 / 2}\left\{\int_{R} P^{2} d A\right\}^{1 / 2}+2^{(1-\delta) / 2} K^{1 / 2}\{E(0)\}^{(1-\delta) / 2} S^{1 / 2}\left\{\int_{R} P^{2 / \delta} d A\right\}^{\delta / 2} \\
& +\left(2 \tau^{2}\right)^{(1-\delta) / 2} K^{1 / 2} S^{(1-\delta) / 2}\left(\int_{R} P^{2} d A\right)^{\delta / 2} .
\end{aligned}
$$

Factoring out $S^{1 / 2}$ we find

$$
\begin{aligned}
S^{1 / 2} \leq & M^{1 / 2}\left\{\int_{R} P^{2} d A\right\}^{1 / 2}+2^{(1-\delta) / 2} K^{1 / 2}\{E(0)\}^{(1-\delta) / 2}\left\{\int_{R} P^{2 / \delta} d A\right\}^{\delta / 2} \\
& +\left(2 \tau^{2}\right)^{(1-\delta) / 2} K^{1 / 2} S^{(1-\delta) / 2}\left(\int_{R} P^{2} d A\right)^{\delta / 2} \\
\leq & M^{1 / 2}\left\{\int_{R} P^{2} d A\right\}^{1 / 2}+2^{(1-\delta) / 2} K^{1 / 2}\{E(0)\}^{(1-\delta) / 2}\left\{\int_{R} P^{2 / \delta} d A\right\}^{\delta / 2} \\
& +\left(2 \tau^{2}\right)^{(1-\delta) / 2} K^{1 / 2}\left\{(1-\delta) \gamma_{1} S^{1 / 2}+\frac{\delta}{\gamma_{1}^{(1-\delta) / \delta}}\left[\int_{R} P^{2} d A\right]^{1 / 2}\right\},
\end{aligned}
$$

where we have used (4.38) in the final step. Here $\gamma_{1}$ is a positive constant at our disposal. If we choose

$$
\gamma_{1}=\frac{1}{2}\left\{\left(2 \tau^{2}\right)^{(1-\delta) / 2} K^{1 / 2}(1-\delta)\right\}^{-1}
$$

and solve for $S^{1 / 2}$ we obtain

$$
\begin{aligned}
S^{1 / 2} \leq & 2\left\{M^{1 / 2}+\left(2 \tau^{2}\right)^{(1-\delta) / 2} K^{1 / 2} \delta\left[2\left(2 \tau^{2}\right)^{(1-\delta) / 2} K^{1 / 2}(1-\delta)\right]^{(1-\delta) / \delta}\right\}\left\{\int_{R} P^{2} d A\right\}^{1 / 2} \\
& +2^{(3-\delta) / 2} K^{1 / 2}\{E(0)]^{(1-\delta) / 2}\left\{\int_{R} P^{2 / \delta} d A\right\}^{\delta / 2} \\
= & C_{1}\left\{\int_{R} P^{2} d A\right\}^{1 / 2}+C_{2}\{E(0)\}^{(1-\delta) / 2}\left\{\int_{R} P^{2 / \delta} d A\right\}^{\delta / 2}
\end{aligned}
$$

where

$$
\begin{aligned}
& C_{1}=2\left\{M^{1 / 2}+\left(2 \tau^{2}\right)^{(1-\delta) / 2} K^{1 / 2} \delta\left[2\left(2 \tau^{2}\right)^{(1-\delta) / 2} K^{1 / 2}(1-\delta)\right]\right\}^{(1-\delta) / \delta} \\
& C_{2}=2^{(3-\delta) / 2} K^{1 / 2}
\end{aligned}
$$


Using the choices for $P^{2}$ suggested by the right-hand side of (B.13) and inserting (B.19) back into (B.13) we conclude that

$$
\begin{aligned}
I_{1} \leq & \{E(0)\}^{1 / 2}\left\{C_{1}\left[\int_{R} w_{, \alpha \beta} w_{, \alpha \beta} d A\right]^{1 / 2}+C_{2}[E(0)]^{(1-\delta) / 2}\left[\int_{R}\left(w_{, \alpha \beta} w_{, \alpha \beta}\right)^{1 / \delta} d A\right]^{\delta / 2}\right\} \\
& +\tau\left\{C_{1}\left[\int_{R}\left|w_{, 22}\right| d A\right]^{1 / 2}+C_{2}[E(0)]^{(1-\delta) / 2}\left[\int_{R}\left|w_{, 22}\right|^{1 / \delta} d A\right]^{\delta / 2}\right\}^{2} .
\end{aligned}
$$

Combining (B.6) and (B.21) and inserting into (B.2) we now arrive at the estimate

$$
\begin{aligned}
& \frac{(1-\sigma)}{2} \int_{0}^{l} E(s) d s \\
& \leq\{E(0)\}^{1 / 2}\left\{C_{1}\left[\int_{R} w_{, \alpha \beta} w_{, \alpha \beta} d A\right]^{1 / 2}+C_{2}[E(0)]^{(1-\delta) / 2}\left[\int_{R}\left(w_{, \alpha \beta} w w_{, \alpha \beta}\right)^{1 / \delta}\right]^{\delta / 2}\right\} \\
& \quad+\tau\left\{C_{1}\left[\int_{R}\left|w_{, 22}\right| d A\right]^{1 / 2}+C_{2}[E(0)]^{(1-\delta) / 2}\left[\int_{R}\left|w_{, 22}\right|^{1 / \delta} d A\right]^{\delta / 2}\right\}^{2} \\
& \quad+C E(0)+D[E(0)]^{(3-\delta) / 2} .
\end{aligned}
$$

Inequality (B.22) is the desired bound for $\int_{0}^{l} E(s) d s$ in terms of $E(0)$. Once we have obtained an explicit bound for $E(0)$ in terms of data we will then have the required bound for $H(0)$ in the case $K \neq 0$. We now derive the bound for $E(0)$.

From (4.17) (with $z=0$ ) we observe that if we introduce an auxiliary function

$$
\begin{aligned}
w^{*}\left(x_{1}, x_{2}\right)= & \left\{F-\frac{\tau}{2} x_{2}^{2}+x_{1}\left[G-\tau x_{2}+2 b\left(F-\frac{\tau x_{2}^{2}}{2}\right)\left(1-e^{-b l}\right)^{-1}\right]\right\} \\
& \times\left[\frac{e^{-b x_{1}}-e^{-b l}}{1-e^{-b l}}\right]^{2}
\end{aligned}
$$

we may equivalently write

$$
\frac{(1-\sigma)}{2} E(0) \leq \int_{R} \rho\left(q^{2}\right) \phi_{, \alpha \beta} w_{, \alpha \beta}^{*} d A,
$$

provided $F$ and $G$ are sufficiently smooth and vanish appropriately on $x_{2}=0$, $x_{2}=h$. Here $b$ is a positive constant at our disposal. To see this we note that $w^{*}$ satisfies

$$
\begin{aligned}
& w^{*}\left(0, x_{2}\right)=\psi\left(0, x_{2}\right), \quad w^{*}{ }_{1}\left(0, x_{2}\right)=\psi_{.1}\left(0, x_{2}\right) \quad\left(0 \leq x_{2} \leq h\right) \\
& w^{*}, w^{*}{ }_{2}=0 \quad \text { on } x_{2}=0, h \quad\left(0 \leq x_{1} \leq l\right), \\
& w^{*}\left(l, x_{2}\right)=w^{*}{ }_{1}\left(l, x_{2}\right)=0 \quad\left(0 \leq x_{2} \leq h\right) .
\end{aligned}
$$

Since the right-hand side of (B.24) is of precisely the same form as $I_{1}$ (see (B.12)) 
with $w$ replaced by $w^{*}$, it follows immediately from (B.21) that

$$
\begin{aligned}
\frac{(1-\sigma)}{2} E(0) \leq\{ & E(0)\}^{1 / 2}\left\{C_{1}\left(\int_{R} w_{, \alpha \beta}^{*} w_{, \alpha \beta}^{*} d A\right)^{1 / 2}\right. \\
& \left.+C_{2}[E(0)]^{(1-\delta) / 2}\left[\int_{R}\left(w_{, \alpha \beta}^{*} w_{, \alpha \beta}^{*}\right)^{1 / \delta} d A\right]^{\delta / 2}\right\} \\
& +\tau\left\{C_{1}\left[\int_{R}\left|w_{, 22}^{*}\right| d A\right]^{1 / 2}+C_{2}[E(0)]^{(1-\delta) / 2}\left[\int_{R}\left|w_{, 22}^{*}\right|^{1 / \delta} d A\right]^{\delta / 2}\right\}^{2} \\
= & {\left[Q_{1}\left(w^{*}\right)\right]^{1 / 2}[E(0)]^{1 / 2}+\left[Q_{2}\left(w^{*}\right)\right]^{\delta / 2}[E(0)]^{1-\delta / 2} } \\
& +Q_{4}\left(w^{*}\right)+\left[Q_{5}\left(w^{*}\right)\right]^{(1+\delta) / 2}[E(0)]^{(1-\delta) / 2}+\left[Q_{6}\left(w^{*}\right)\right]^{\delta}[E(0)]^{1-\delta},
\end{aligned}
$$

with the obvious definitions of the $Q_{i}$ 's. Repeated use of (4.39) in the last expression in (B.26) leads to

$$
\begin{aligned}
& \left\{\frac{(1-\sigma)}{2}-\frac{\gamma_{2}}{2}-\gamma_{3}\left(1-\frac{\delta}{2}\right)-\gamma_{4}\left(\frac{1-\delta}{2}\right)-\gamma_{5}(1-\delta)\right\} E(0) \\
& \leq \frac{1}{2 \gamma_{2}} Q_{1}\left(w^{*}\right)+\frac{\delta}{2 \gamma_{3}^{(2-\delta) / \delta}} Q_{2}\left(w^{*}\right)+Q_{4}\left(w^{*}\right)+\frac{1+\delta}{2 \gamma_{4}^{(1-\delta) /(1+\delta)}} Q_{5}\left(w^{*}\right) \\
& \quad+\frac{\delta}{\gamma_{5}^{(1-\delta) / \delta}} Q_{6}\left(w^{*}\right)
\end{aligned}
$$

for appropriate choices of the $\gamma_{i}$ 's. For instance the choice

$$
\gamma_{2}=\frac{1-\sigma}{16}, \quad \gamma_{3}=\frac{(1-\sigma)}{8(2-\delta)}, \quad \gamma_{4}=\frac{1-\sigma}{8(1-\delta)}, \quad \gamma_{5}=\frac{1-\sigma}{16(1-\delta)}
$$

leads to

$$
\begin{aligned}
\frac{(1-\sigma)}{4} E(0) \leq & \frac{8}{1-\sigma} Q_{1}\left(w^{*}\right)+\frac{\delta}{2}\left[\frac{8(2-\delta)}{1-\sigma}\right]^{(2-\delta) / \delta} Q_{2}\left(w^{*}\right)+Q_{4}\left(w^{*}\right) \\
& +\frac{1-\delta}{2}\left[\frac{8(1-\delta)}{1-\sigma}\right]^{(1-\delta) /(1+\delta)} Q_{5}\left(w^{*}\right)+\delta\left[\frac{16(1-\delta)}{1-\sigma}\right]^{(1-\delta) / \delta} Q_{6}\left(w^{*}\right),
\end{aligned}
$$

which is the desired explicit bound for $E(0)$ in the case $K \neq 0$. The insertion of (B.29) into (B.22) yields an explicit bound for $\int_{0}^{l} E(s) d s$ and the combination of the two gives finally the desired bound for $H(0)$ in the case $K \neq 0$.

When the constant $K$ in (4.3) is zero, i.e., when $\rho$ is not only bounded away from zero, but also bounded above, we can read off the results from our previous computations. As was marked at the beginning of Appendix B, the decay estimate (4.13) for $E(z)$ is explicit in this case once we have a bound for $E(0)$. Making use of the fact that the bound for $(1-\sigma) E(0) / 2$ in (B.24) has the same form as $I_{1}$ in (B.12) with $w$ replaced by $w^{*}$, and using (B.21) (with $K=0$ so that by (B.20) $\left.C_{1}=2 M^{1 / 2}, C_{2}=0\right)$ we find

$$
\frac{(1-\sigma)}{2} E(0) \leq 2 M^{1 / 2}\left\{\int_{R} w^{*}{ }_{, \alpha \beta} w^{*}{ }_{, \alpha \beta} d A\right\}^{1 / 2}\{E(0)\}^{1 / 2}+4 M \tau \int_{R}\left|w^{*}{ }_{.22}\right| d A .
$$


An application of the arithmetic-geometric mean inequality on the first term on the right now leads to

$$
\frac{(1-\sigma)}{4} E(0) \leq 4 M\left\{\frac{1}{1-\sigma} \int_{R} w_{, \alpha \beta}^{*} w_{, \alpha \beta}^{*} d A+\tau \int_{R}\left|w_{, 22}^{*}\right| d A\right\} .
$$

The insertion of (B.31) into (4.113) then yields an explicit decay inequality in the case $K=0$.

We remark that the constants $a$ and $b$, introduced in (B.7) and (B.23) respectively, are still arbitrary. Ideally, one would like to choose these constants to minimize the bounds on $E(0)$ (when $K=0$ ) and on $H(0)$ (when $K \neq 0$ ). However, any choice for $a, b \quad(>0)$ will yield explicit bounds on these quantities.

Acknowledgments. The work of C. O. H. was supported by the U. S. National Science Foundation (NSF) under Grant No. MSS-91-02155, by the U. S. Air Force Office of Scientific Research under Grant No. AFOSR-F49620-92-J-0112, and by the U. S. Army Research Office under Grant No. DAAL03-91-G-0022. The work of L. E. P. was carried out while he held an appointment as Visiting Professor, Department of Applied Mathematics, University of Virginia, Spring, 1990, and was partially supported by the NSF under Grant No. DMS-91-00786.

\section{REFERENCES}

[1] C. O. Horgan and J. K. Knowles, The effect of nonlinearity on a principle of Saint-Venant type, J. Elasticity 11, 271-291 (1981)

[2] C. O. Horgan and J. K. Knowles, Recent developments concerning Saint-Venant's principle, Advances in Applied Mechanics (J. W. Hutchinson, ed.), Vol. 23, Academic Press, New York, 1983, pp. 179-269

[3] C. O. Horgan, Recent developments concerning Saint-Venant's principle: an update, Appl. Mech. Rev. 42, 295-303 (1989)

[4] G. Fichera, Il principio di Saint-Venant: Intuizione dell' ingegnere e rigore del matematico, Rend. Mat. (6) 10, 1-24 (1977)

[5] G. Fichera, Remarks on Saint-Venant's principle, Rend Mat. (6) 12, 181-200 (1979)

[6] J. L. Ericksen, On the formulation of St. Venant's problem, Nonlinear Analysis and Mechanics: Heriot-Watt Symposium (R. J. Knops, ed.), Vol. I, Pitman, London, 1977, pp. 158-186

[7] R. G. Muncaster, Saint-Venant's problem in nonlinear elasticity: a study of cross-sections, Nonlinear Analysis and Mechanics: Heriot-Watt Symposium (R. J. Knops, ed.), Vol. IV, Pitman, London, 1979, pp. 17-75

[8] R. G. Muncaster, Saint-Venant's problem for slender prisms, Utilitas Math. 23, 75-101 (1983)

[9] J. L. Ericksen, Saint-Venant's problem for elastic prisms, Systems of Nonlinear Partial Differential Equations (J. M. Ball, ed.), D. Reidel, Dordrecht, 1983, pp. 87-93

[10] J. L. Ericksen, Problems for infinite elastic prisms, Systems of Nonlinear Partial Differential Equations (J. M. Ball, ed.), Reidel, Dordrecht, 1983, pp. 80-86

[11] D. Kinderlehrer, A relation between semi-inverse and Saint-Venant solutions for prisms, SIAM J. Math. Anal. 17, 626-640 (1986)

[12] D. Kinderlehrer, Remarks about Saint-Venant solutions in finite elasticity, Nonlinear Functional Analysis and its Applications: Proceedings of Symposia in Pure Mathematics (F. E. Browder, ed.), Vol. 45, Part 2, Amer. Math. Soc., Providence, RI, 1986, pp. 37-50

[13] A. Mielke, Saint-Venant's problem and semi-inverse solutions in nonlinear elasticity, Arch. Rational Mech. Anal. 102, 205-229 (1988)

[14] S. Breuer and J. J. Roseman, On Saint-Venant's principle in three-dimensional nonlinear elasticity, Arch. Rational Mech. Anal. 63, 191-203 (1977)

[15] S. Breuer and J. J. Roseman, Saint-Venant's principle in nonlinear plane elasticity with sufficiently small strains, Arch. Rational Mech. Anal. 80, 19-37 (1982) 
[16] R. J. Knops and L. E. Payne, A Saint-Venant principle for nonlinear elasticity, Arch. Rational Mech. Anal. 81, 1-12 (1983)

[17] C. O. Horgan and R. Abeyaratne, Finite anti-plane shear of a semi-infinite strip subject to a selfequilibrated end traction, Quart. Appl. Math. 40, 407-417 (1983)

[18] G. P. Galdi, R. J. Knops, and S. Rionero, Asymptotic behavior in the nonlinear elastic beam, Arch. Rational Mech. Anal. 87, 305-318 (1985)

[19] R. Abeyaratne, C. O. Horgan, and D.-T. Chung, Saint-Venant end effects for incremental plane deformations of incompressible nonlinearly elastic materials, J. Appl. Mech. 52, 847-852 (1985)

[20] A. Mielke, Normal hyperbolicity of center manifolds and Saint-Venant's principle, Arch. Rational Mech. Anal. 110, 353-372 (1990)

[21] D. Durban and W. J. Stronge, Diffusion of self-equilibrating end loads in elastic solids, J. Appl. Mech. 5, 492-495 (1988)

[22] P. Vafeades and C. O. Horgan, Exponential decay estimates for solutions of the von Kármán equations on a semi-infinite strip, Arch. Rational Mech. Anal. 104, 1-25 (1988)

[23] R. Quintanilla, Asymptotic behavior of solutions in elasticity, preprint, Univ. Polit. de Catalunya, Barcelona, Spain, 1987

[24] H. A. Levine and R. Quintanilla, Some remarks on Saint-Venant's principle, Math. Methods Appl. Sci. 11, 71-77 (1989)

[25] B. B. Orazov, On the asymptotic behavior at infinity of solutions of the traction boundary value problem, Proc. Roy. Soc. Edinburgh Sec. A 111, 33-52 (1989)

[26] C. O. Horgan and L. E. Payne, On Saint-Venant's principle in finite anti-plane shear: an energy approach, Arch. Rational Mech. Anal. 109, 107-137 (1990)

[27] C. O. Horgan, L. E. Payne, and J. G. Simmonds, Existence, uniqueness and decay estimates for solutions in the nonlinear theory of elastic, edge-loaded, circular tubes, Quart. Appl. Math. 48, 341-359 (1990)

[28] C. O. Horgan and L. E. Payne, Decay estimates for second-order quasilinear partial differential equations, Adv. in Appl. Math. 5, 309-332 (1984)

[29] C. O. Horgan and L. E. Payne, Decay estimates for a class of second-order quasilinear equations in three dimensions, Arch. Rational Mech. Anal. 86, 279-289 (1984)

[30] C. O. Horgan, A note on the spatial decay of a three-dimensional minimal surface over a semiinfinite cylinder, J. Math. Anal. Appl. 107, 285-290 (1985)

[31] S. Breuer and J. J. Roseman, Phragmén-Lindelöf decay theorems for classes of nonlinear Dirichlet problems in a circular cylinder, J. Math. Anal. Appl. 113, 59-77 (1986)

[32] S. Breuer and J. J. Roseman, Decay theorems for nonlinear Dirichlet problems in semi-infinite cylinders, Arch. Rational Mech. Anal. 94, 363-371 (1986)

[33] C. O. Horgan, Some applications of maximum principles in linear and nonlinear elasticity, Maximum Principles and Eigenvalue Problems in Partial Differential Equations (P. W. Schaefer, ed.), Pitman Res. Notes Math. Ser., vol. 175, Longman, New York, 1988, pp. 49-67

[34] C. O. Horgan and L. E. Payne, Decay estimates for a class of nonlinear boundary value problems in two dimensions, SIAM J. Math. Anal. 20, 782-788 (1989)

[35] C. O. Horgan and L. E. Payne, On the asymptotic behavior of solutions of inhomogeneous secondorder quasilinear partial differential equations, Quart. Appl. Math. 47, 753-771 (1989)

[36] S. Breuer and J. J. Roseman, On spatial energy decay for quasilinear boundary value problems in cone-like and exterior domains, Differential Integral Equations 2, 310-325 (1989)

[37] J. J. Roseman and S. Zimering, On the spatial decay of the energy for some quasilinear boundary value problems in semi-infinite cylinders, J. Math. Anal. Appl. 139, 194-204 (1989)

[38] C. O. Horgan and D. Siegel, On the asymptotic behavior of a minimal surface over a semi-infinite strip, J. Math. Anal. Appl. 153, 397-406 (1990)

[39] R. Quintanilla, Exponential decay for nonlinear second-order partial differential equations, preprint, Univ. Polit. de Catalunya, Barcelona, Spain, 1990

[40] G. H. Hardy, J. E. Littlewood, and G. Pólya, Inequalities, Cambridge Univ. Press, Cambridge, 1964

[41] C. O. Horgan and W. E. Olmstead, Exponential decay estimates for a class of nonlinear Dirichlet problems, Arch. Rational Mech. Anal. 71, 221-235 (1979)

[42] J. J. Roseman, The principle of Saint-Venant in linear and nonlinear plane elasticity, Arch. Rational Mech. Anal. 26, 142-162 (1967) 